\title{
Prevention of pressure oscillations in modeling a cavitating acoustic fluid
}

\author{
B. Klenow* and A. Brown \\ Department of Aerospace and Ocean Engineering, Virginia Polytechnic Institute and State University, Blacksburg, \\ VA 24061, USA
}

Received 3 September 2008

Revised 12 April 2009

\begin{abstract}
Cavitation effects play an important role in the UNDEX loading of a structure. For far-field UNDEX, the structural loading is affected by the formation of local and bulk cavitation regions, and the pressure pulses resulting from the closure of the cavitation regions. A common approach to numerically modeling cavitation in far-field underwater explosions is Cavitating Acoustic Finite Elements (CAFE) and more recently Cavitating Acoustic Spectral Elements (CASE). Treatment of cavitation in this manner causes spurious pressure oscillations which must be treated by a numerical damping scheme. The focus of this paper is to investigate the severity of these oscillations on the structural response and a possible improvement to CAFE, based on the original Boris and Book Flux-Corrected Transport algorithm on structured meshes [6], to limit oscillations without the energy loss associated with the current damping schemes.
\end{abstract}

\section{Introduction}

For the past 20 years the finite element method (FEM) has been frequently used to model far-field underwater explosion (UNDEX) phenomena [12,13,18,26-28,30,33,34]. A far-field, or distant shot, underwater explosion is defined here as an UNDEX where the charge is located at a sufficient distance away from the structure such that effects from the explosive gas bubble are negligible. For the purposes of the acoustic fluid models used in this work we also assume the structural response to the far-field explosion to be elastic.

Far-field underwater explosions are of particular interest because they are the type of UDNEX used in Navy ship shock trials (see e.g. [31]). An important goal of applying FEM to far-field UNDEX problems is to accurately simulate full scale ship shock trials to aid in the design of new naval ships [30].

A FE model of a far-field UNDEX generally consists of a structure domain, a fluid domain, and a fluid boundary which is treated as a non-reflecting boundary. The far-field UNDEX model must consider two UNDEX phenomena, the shock wave that results from the detonation of explosive material, and cavitation. It is important to note that typically a far-field FE model does not explicitly include the charge. Instead the initial shock wave incident on the structure is initialized inside the fluid, close to the structure. Cavitation results from the pressure in the surrounding water dropping below its vapor pressure due to the motion of the ship structure (local cavitation) or the tensile shock wave reflected from the free surface (bulk cavitation).

Both the incident shock wave and the cavitation regions present computational difficulties in far-field UNDEX finite element models. The incident shock wave is a discontinuous wave, making the numerical dispersion of the wave front and dissipation of pressure magnitude a concern. Cavitation creates a material discontinuity in the fluid and may cause a secondary shock loading upon closure. The formation of cavitation regions is also affected by dispersion and dissipation of the reflected shock wave. Dispersion and dissipation of the incident, reflected, and

\footnotetext{
*Corresponding author. Tel.: +1 540231 9472; Fax: +1 540231 9632; E-mail: klenowb@vt.edu.
} 
cavitation closure shock waves as well as the discontinuous nature of cavitation typically require FE models of far-field UNDEX to have fine meshes for accurate solutions [33,34].

The need for fine meshes in far-field UNDEX FE models is a major obstacle in the ability to accurately simulate ship shock trials because far-field UNDEX models require a substantial amount of fluid to be modeled, especially in a full scale three-dimensional problem. In these types of problems the size requirement of the fluid model and the need for a fine mesh cause the computational effort to be extreme [34,35].

The size requirements of the fluid domain and the far-field fluid/structure assumptions make treating the fluid as an acoustic fluid with scalar unknowns a popular choice in far-field UNDEX FE models. An acoustic fluid is one in which disturbances in the fluid propagate at a constant speed and are small (geometrically linear), even if the constitutive behavior (i.e., cavitation phenomena) of the fluid is non-linear [12]. Such a treatment is valid in the farfield problem, because shock and cavitation loading are early time events, occurring on the order of micro-seconds. Because of the short time duration of these early time events, the ship as whole does not exhibit a significant global response to the loading. Furthermore the late time effects of the gas bubble and hull rupture, two phenomena that cause large fluid displacements, can be neglected. Thus the early time far-field problem is of short duration and the global fluid displacement is small. This satisfies the conditions for acoustic treatment given in [43].

\section{Far-field UNDEX fluid modeling}

\subsection{Cavitating acoustic finite elements}

Newton $[26,27]$ developed one of the first FEM cavitation models using the displacement potential formulation of the acoustic wave equation. In his cavitation model a bilinear constitutive equation, based on the work of Bleich and Sandler [3], is used to treat the cavitation region as a homogenous single phase region of constant total pressure equal to the vapor pressure of water. In most models the vapor pressure of water is taken to be 0 Pa because the large difference between the magnitude of vapor pressure, $\sim 10^{3} \mathrm{~Pa}$, and hydrostatic pressure, $\sim 10^{5} \mathrm{~Pa}$, negates any significance for using the actual vapor pressure in the cavitation region $[13,34]$.

This type of cavitation model is a one-fluid, cut-off cavitation model [39]. One-fluid refers to the single governing equation used to model two separate phases of the fluid. The advantage of a one-fluid model is computational savings, especially in large scale problems, compared to a two-fluid model which requires multiple constitutive fluid equations to be solved [39].

Cut-off refers to the method by which the cavitation model forces the total pressure of the fluid to equal the vapor pressure of water once the cavitation criterion is met. The disadvantage of the cut-off treatment is that the phase transition between water and vapor is not explicitly considered. However, in large scale problems, such as a far-field UDNEX, cut-off models have performed almost identically to cavitation models that include phase transition [22, 40].

Newton's cavitation model was used by Zienkiewicz et al. [44] to study cavitation effects on dam loading. In their model an unconditionally stable implicit time integration scheme was introduced, with an iterative check for cavitation. Felippa and DeRuntz [12,13] latter used Newton's cavitation model in the development of the Cavitating Fluid Analysis (CFA) code. The CFA code extended Newton's model to three dimensions, incorporated a conditionally stable staggered central difference time integration scheme, a node-by-node (non-iterative) check for cavitation, and coupled the fluid boundary to the boundary element code USA to act as a non-reflecting boundary. This approach has been incorporated into the commercial FEM code LS-DYNA [18,34] and has been used by many researchers in far-field UNDEX modeling applications [18,19,31,32,37]. Sprague and Geers [32] called the approach created by Felippa and DeRuntz as Cavitating Acoustic Finite Elements (CAFE) and we adopt this notation in our work.

In $[34,35]$ Sprague and Geers detail several aspects of the CAFE approach that limit its effectiveness, especially for large scale problems. The first deficiency in the CAFE approach is the use of linear (1D), bi-linear (2D), or tri-linear (3D) elements in the fluid mesh. Because low order elements are used, CAFE fluid meshes are highly dispersive [34,35]. This makes the CAFE approach very dependent on the mesh refinement discussed in the previous section. Such a high level of CAFE mesh refinement is often not feasible for full ship far-field UNDEX models. 
Additionally, in the implementation of CAFE in [12,13], the structural model is solved implicitly which is less computationally efficient than explicit methods. Another deficiency of the implementation of the CAFE approach in $[12,13]$ is that one-to-one nodal coupling is required at every point on the fluid-structure interface. The one-to-one coupling requirement makes it extremely difficult to couple a complex structure to the fluid. In addition, one-to-one coupling requires that any mesh refinement in the fluid must also exist in the structural elements on the fluid-structure interface [34]. Such structural refinements are not usually necessary, and when the structure is solved explicitly can cause a decrease in the critical time step of the model because of the differences in material sound speeds (with that in steel being substantially greater than that in water).

\subsection{Cavitating acoustic spectral elements}

To address the highly dispersive nature of the CAFE approach, Sprague and Geers employed higher order spectral elements (SE) to discretize the governing CAFE equations [34-36]. They called this approach Cavitating Acoustic Spectral Elements (CASE).

Spectral elements are a combination of spectral methods and FEM. Spectral methods use higher order basis functions derived from orthogonal polynomials to reduce interpolation error at the integration points or nodes. Typically either Chebyshev or Legendre polynomials are used for the orthogonal polynomials that define the basis functions in the spectral element method (SEM). Sprague and Geers prefer Legendre polynomials because of their superior performance in wave propagation problems [35] and use Gauss-Lobatto-Legendre (GLL) quadrature for the node/interpolation points.

High order spectral elements are generally less dispersive than low order elements [25,34] and can exhibit exponential convergence for problems with smooth solutions [29,34]. When combined with FEM, the spectral basis functions are applied to each element using a sub-parametric mapping, rather than to the entire domain. The result is a method that retains the geometric flexibility of FEM and the high order accuracy of spectral methods [35].

The application of spectral elements in the CASE approach improves the dispersion and refinement issues encountered in the CAFE approach. The result is a savings in required degrees of freedom, which in turn reduces total operations and memory storage $[34,36]$. In their implementation of CASE Sprague and Geers also incorporated field separation and a constant-interpolation coupling [11] algorithm to couple the fluid to the structure. The coupling algorithm removes the need for one-to-one nodal coupling along the fluid-structure interface, making coupling complex structures to the fluid easier and also allowing the fluid mesh to be refined separately from the structural mesh [35]. Field separation separates the fluid into a known incident field (the incident shock wave) and the unknown scattered field (the reflected shock and cavitation region) which are combined to obtain the total field. The advantage of field separation is that the incident shock wave can be propagated through the mesh without being distorted [34, 35].

Despite the advantages of the CASE approach there are deficiencies that limit its effectiveness in certain problems. One disadvantage is that spectral elements require smaller critical time steps for explicit time integration [34,35]. An important implication of the reduced critical time step is that when compared to a CAFE model with the same number of total degrees of freedom, a CASE model is more computationally expensive.

The most significant disadvantage of the CASE approach is the tendency of spectral elements to increase spurious oscillations in wave propagation problems [25]. This is especially true for problems with discontinuities $[15,16,36]$, such as the incident and reflected shock wave and the cavitation region boundaries encountered in UNDEX problems. In CAFE and CASE models of UNDEX increased oscillations are problematic due to the formation of cavitation regions. Oscillations due to cavitation formation in the CAFE approach were initially discussed by Newton. Newton observed that small isolated regions of caviated fluid can appear within the pressurized fluid and that small isolated regions of pressurized fluid can appear within a region of cavitation [26,27]. He called this phenomenon "frothing". In $[34,35]$, CASE models exhibited larger oscillations in the structural response due to an increase in frothing in the fluid domain. We have observed that frothing occurs in the pressurized fluid that exists between the upper cavitation boundary and the structure/free surface. The absence of frothing does not mean oscillations have been sufficiently damped, only that the magnitude of the oscillations has not reached the cavitation cut-off condition. These oscillations are unique to cavitation problems because they form from a combination of discontinuous wave propagation (the reflected wave front), a material discontinuity in the fluid (cavitation region boundaries), and the application of the cavitation model itself. 


\section{Finite element flux-corrected transport}

\subsection{Flux-corrected transport overview}

Given the advantages and disadvantages of both the CAFE and CASE approaches we sought an improvement that combines the less diffusive nature of CASE with the less oscillatory nature and reduced computational expense of CAFE. To accomplish this we evaluated a finite element flux-corrected transport (FE-FCT) method proposed by Xiao [38] for structured grids. The flux-corrected transport method was developed by Boris and Book [5-7] to predict smooth finite difference solutions to one-dimensional shock problems in compressible fluids governed by the continuity and/or Euler equations. In general, a flux-corrected transport algorithm consists of a transported diffusion stage and an anti-diffusion stage. The transported diffusion stage advances the solution in time and gives a smooth or diffused solution. The anti-diffusion stage seeks to correct the numerical errors introduced by the diffused solution by correcting or limiting the anti-diffusive fluxes [38].

Zalesak [41,42] extended flux limitation and FCT to a general multi-dimensional form by using the combination of a high and low order numerical scheme. The low order scheme, like the diffusion stage of the original algorithm, gives a smooth solution in regions of discontinuity. The high order scheme gives a more accurate solution in smooth regions of the solution. The FCT algorithm developed by Zalesak has been applied to many problems involving shocks in fluids. Similar FCT algorithms have been developed for FEM [14,24] and even SEM [15,16].

For far-field UNDEX problems the FE-FCT algorithm introduced by Xiao is ideal because it does not require the combination of a high and low order scheme. Use of a high and low order scheme would be too computationally expensive for full scale far-field UNDEX problems. FE-FCT is a well suited method for application to CAFE like methods because it both smoothes the solution, which is needed to capture cavitation, and restores accuracy, which is needed to capture the initial and reflected shock wave. Another beneficial attribute of FE-FCT algorithms for UNDEX applications is that they are typically less diffusive than artificial damping schemes. This is important for CAFE/CASE methods because the amount of artificial damping needed to adequately smooth cavitation oscillations often over damps the energy associated with shock wave propagation.

\subsection{FE-FCT algorithm}

A general representation of the FCT algorithm devised by Boris and Book [5,6] for finite difference methods and extended to the finite element method by Xiao [38] is given below. Following Xiao's routine, let $\psi$ be a function of time and space in one-dimension, whose value at time step $n+1$ and location $x$ (represented by nodal index $j$ ) is a function of the value of $\psi_{j}$ at time step $n$, time step $\Delta t$, and element length $\Delta h$. Xiao's FE-FCT algorithm then consists of the following steps:

1. Compute transported solution by FE approximation of $\psi_{j}$ at time step $n+1$ :

$$
\tilde{\psi}_{j}^{n+1}=f\left(\psi_{j}^{n}, \Delta t, \Delta h\right) .
$$

2. Compute the diffusive fluxes:

$$
\varphi_{j}^{D}=\eta_{D}\left(\psi_{j+1}^{n}-\psi_{j}^{n}\right),
$$

where $\eta_{D}$ is the diffusion coefficient.

3. Compute the transported diffused solution:

$$
\psi_{j}^{T D}=\tilde{\psi}_{j}^{n+1}+\varphi_{j}^{D}-\varphi_{j-1}^{D} .
$$

4. Compute the anti-diffusive fluxes:

$$
\varphi_{j}^{A}=\eta_{A}\left(\tilde{\psi}_{j+1}^{n}-\tilde{\psi}_{j}^{n}\right)
$$

where $\eta_{A}$ is the anti-diffusion coefficient.

5. Limit/correct the anti-diffusive fluxes:

$$
\varphi_{j}^{c}=S \cdot \operatorname{mas}\left\{0, \min \left(S \cdot \Delta_{j-1},\left|\varphi_{j}^{A}\right|, S \cdot \Delta_{j+1}\right)\right\},
$$

where $\Delta_{j \pm 1}= \pm\left(\psi_{j \pm 1}^{T D}-\psi_{j}^{T D}\right)$ and $S=\operatorname{sign}\left(\varphi_{j}^{A}\right)$. 
6. Apply the corrected anti-diffusive fluxes to the solution:

$$
\psi_{j}^{n+1}=\psi_{j}^{T D}+\varphi_{j}^{c}-\varphi_{j-1}^{c} .
$$

The diffusive, $\eta_{D}$, and anti-diffusive, $\eta_{A}$, coefficients are numerical parameters, chosen to suit a particular problem or algorithm. We discuss the selection of these parameters in Section 4.3. The key component of the FCT algorithm is the limiting of the anti-diffusive fluxes in Eq. (5). This limiter, which is referred to as a strong flux limiter [4], allows the addition of anti-diffusion to the solution, which allows errors introduced by the diffusive stage of the algorithm to be corrected, without allowing existing oscillations to grow or causing new oscillations [6].

\section{FE-FCT for far-field UNDEX}

\subsection{Governing fluid equations}

For application to the FE-FCT scheme outline in the previous section we use the pressure form of the acoustic wave equation. We assume that viscosity and convective acceleration terms are negligible and that the reference density of the fluid, $\rho_{o}$, is constant and that fluctuations between the density, $\rho$, and $\rho_{o}$ remain small. This allows the relation between condensation, $s=\frac{\rho}{\rho_{o}}-1$, and dynamic pressure, $p$, to be taken as [20]

$$
\rho_{o} \frac{\partial^{2} s}{\partial t^{2}}=\nabla^{2} p
$$

where $|s|<<1$.

In [27], Nimmagadda and Cipolla derived a cavitation model for Eq. (7). This cavitation model coupled with the pressure form of the AWE are used in the commercial finite element code ABAQUS [2]. Both [2,28] define a "pseudo-pressure" as the material state variable instead of condensation (as in CAFE/CASE). Following their approach, the pseudo-pressure is given by

$$
p_{v}=-p_{o} c^{2}(\nabla \cdot \vec{\xi})
$$

where $c$ is the speed of sound in the fluid and $\vec{\xi}$ is the fluid displacement field. Replacing $s$ in Eq. (7) by the pseudo-pressure given in Eq. (8), the cavitating form of Eq. (7) becomes

$$
\frac{1}{c^{2}} \frac{\partial^{2} p_{v}}{\partial t^{2}}=\nabla^{2} p
$$

The equation of state for $p$ is now determined by the cavitation model

$$
p=\max \left(-p_{h}, p_{v}\right),
$$

where $p_{h}$ is the hydrostatic pressure. Note that the cavitation model in Eq. (10) is a one-fluid cut-off model that satisfies the condition that the total pressure $\left(p_{t}=p+p_{h}\right)$ in the cavitation region is zero.

\subsection{Finite element formulation}

We discretize the governing fluid equation Eq. (9) using the Galerkin approach. Pre-multiplying Eq. (9) by test function $\phi$ and integrating over the fluid domain $\Omega$ gives

$$
\int_{\Omega}\left[\frac{1}{c^{2}} \frac{\partial^{2} p_{v}}{\partial t^{2}} \phi+\nabla^{2} p \phi\right] d \Omega=0 .
$$

Applying Green's first identity to Eq. (11) yields the weak form of the governing equation

$$
\frac{1}{c^{2}} \int_{\Omega} \ddot{p}_{v} \phi d_{\Omega} \int_{\Omega} \nabla p \cdot \nabla \phi d \Omega=\int_{\Gamma} \phi \nabla p \cdot \vec{n} d \Gamma,
$$

where $\Gamma$ denotes the fluid boundary in domain $\Omega$ and $\vec{n}$ is the outward pointing normal vector on $\Gamma$. We approximate the pseudo pressure, dynamic pressure, and test function in Eq. (12) using piecewise linear basis functions, which 
gives the matrix equation

$$
\boldsymbol{Q} \ddot{p}_{v}+\boldsymbol{H} p=\boldsymbol{b},
$$

where $\boldsymbol{Q}$ is the capacitance matrix, $\boldsymbol{H}$ is the reactance matrix, and $\boldsymbol{b}$ is the fluid force vector. The integrals in Eq. (12) are found using Gauss quadrature. A critical aspect of Xiao's FE-FCT scheme is that the algorithm corrects each corrected variable separately. This implies that each corrected variable must be independent, which makes the FE-FCT scheme explicit. Therefore, the capacitance matrix $Q$ must be lumped. This is done using the row-sum technique.

\subsubsection{Boundary conditions}

For far-field UNDEX there are three main types of boundaries that are applied to Eq. (12): known pressure, fluid-structure interaction, and non-reflecting boundaries (NRBC). Known pressure boundaries are boundaries where $p$ is known for all time. A key known pressure boundary for far-field UNDEX is the fluid free surface boundary condition [43]

$$
p=0 .
$$

On fluid-structure interaction boundaries we assume that the fluid and structure have the same displacements and for the purposes of this work we also take the fluid and structure nodes on the fluid-structure boundary to be coincident and leave the incorporation of a more sophisticated coupling method for a later time. Recall that this is the approach taken in the original CAFE method. The fluid-structure interaction boundary condition for Eq. (12) is [2]

$$
\vec{n}_{s} \cdot \vec{a}_{s}=-\frac{1}{\rho_{o}} \frac{\partial p}{\partial n}
$$

where $\vec{n}_{s}$ is the normal vector on the fluid-structure boundary pointing into the fluid in direction $n$ and $\vec{a}_{s}$ is the structural acceleration field on the fluid-structure boundary. This boundary condition is used to determine the fluid force vector $\boldsymbol{b}$.

Non-reflecting boundaries allow the modeled fluid domain, which is finite, to be treated numerically as an infinite domain [17]. Such treatment is necessary for far-field UNDEX problems because physically the fluid region it is part of a large body of water [21]. For this work we apply the Sommerfeld NRBC [16] given in Eq. (16), which is exact for one-dimensional plane waves.

$$
\frac{\partial p}{\partial n}=-\frac{1}{c} \frac{\partial p}{\partial t}
$$

Application of Eq. (16) to Eq. (12) yields a new matrix equation [2]

$$
\boldsymbol{Q} \ddot{p}_{v}+\boldsymbol{D} \dot{p}_{v}+\boldsymbol{H} p=\boldsymbol{b},
$$

where $\boldsymbol{D}$ is a diagonal "damping" matrix that has non-zero terms, $-\frac{1}{c}$, only on NRBC boundary nodes [16]. While the Sommerfeld NRBC is easy to implement and works well in one-dimension, more complex NRBCs are required for accurate solutions to more realistic UNDEX problems. ABAQUS [2] uses the curved wave approximation which was latter implemented by Sprague and Geers [34]. Felippa and DeRuntz used the doubly asymptotic approximation (DAA) via the boundary element code USA $[10,12,13]$ for their implementation of CAFE.

\subsection{Application of FE-FCT algorithm to the acoustic wave equation}

We now apply Xiao's FE-FCT algorithm to the cavitating acoustic formulation given in discretized form by Eqs (12) and (17) and subject to the boundary conditions described above. To predict the rate of change of pressure we apply an explicit time integration algorithm given in [8]. This algorithm has the advantage of centering the Sommerfeld radiation condition in time to preserve the second order accuracy of the algorithm. Also note the following application is for a one-dimensional problem, but it can also be applied to a multidimensional problem where the discontinuous wave is a plane wave. In this case $\dot{p}_{v, i}$ would be corrected in the direction of wave propagation only. Further extension of this algorithm to multiple dimensions for general cases is provided in $[4,38]$.

In the following equations $n$ is the time step number and $i$ is a nodal index: 
1. Compute the transported via the explicit scheme given in [8]:

$$
\tilde{p}_{v}^{n+1 / 2}=\left[\Delta t\left(\boldsymbol{b}^{n}-\boldsymbol{H} p^{n}\right)+\left(\boldsymbol{Q}-\frac{\Delta t}{2} \boldsymbol{D}\right) \dot{p}_{v}^{n-1 / 2}\right] \tilde{\boldsymbol{Q}}^{-1} .
$$

where,

$$
\tilde{\boldsymbol{Q}}=\boldsymbol{Q}+\frac{\Delta t}{2} \boldsymbol{D}
$$

2. Compute the diffusive fluxes:

$$
\varphi_{i}^{D}=\eta_{D}\left(\dot{p}_{v, i+1}^{n-1 / 2}-\dot{p}_{v, i}^{n-1 / 2}\right)
$$

3. Compute the transported diffused solution:

$$
\dot{p}_{v, i}^{T D}=\tilde{p}_{v, i}^{n+1 / 2}+\varphi_{i}^{D}-\varphi_{i-1}^{D} .
$$

4. Compute the anti-diffusive fluxes:

$$
\varphi_{i}^{A}=\eta_{A}\left(\tilde{p}_{v, i+1}^{n-1 / 2}-\tilde{p}_{v, i}^{n-1 / 2}\right),
$$

5. Limit/correct the anti-diffusive fluxes:

$$
\varphi_{i}^{c}=S \cdot \max \left\{0, \min \left(S \cdot \Delta_{i-1},\left|\varphi_{i}^{A}\right|, S \cdot \Delta_{i+1}\right)\right\},
$$

where $\Delta_{i \pm 1}= \pm\left(\dot{p}_{v, i \pm 1}^{T D}-\dot{p}_{v, i}^{T D}\right)$ and $S=\operatorname{sign}\left(\varphi_{j}^{A}\right)$.

6. Apply the corrected anti-diffusive fluxes to the solution:

$$
\dot{p}_{v, i}^{n+1 / 2}=\dot{p}_{v, i}^{T D}+\varphi_{i}^{c}-\varphi_{i-1}^{c} .
$$

7. Update pseudo pressure (Eq. (31)) and check for cavitation using Eq. (16) on each node:

$$
p_{v}^{n+1}=p_{v}^{n}+\Delta t \dot{p}_{v}^{n+1 / 2}
$$

8. Apply known pressure boundary conditions and advance to next time step.

As mentioned in Section 4.1, the diffusive, $\eta_{D}$, and anti-diffusive, $\eta_{A}$, coefficients are numerical parameters. Boris and Book gave $\eta_{D}=\eta_{A}=1 / 8$ [4,6] as the optimal choice of coefficients. These same coefficients were also used by Xiao. While there are no strict rules for choosing the coefficients, in general, $\eta_{D} \geqslant \eta_{A}$, to prevent the growth of new oscillations in the anti-diffusion stage. For the anti-diffusion stage to fully correct the errors introduced in the diffusion stage, $\eta_{D}=\eta_{A}$. This last requirement is problematic for cavitation problems. In order to ensure a smooth solution after the anti-diffusion stage, the diffusion stage must remove all oscillations [41]. Because spurious oscillations are inherent to our cavitation scheme, we cannot ensure that the diffusion stage adequately smoothes the solution. Therefore, for a cavitation problem the solution will retain oscillations after the anti-diffusion stage as the flux limiter. Equation (23) no longer guarantees the formation of new oscillations or the non-growth of existing oscillations.

A solution to this problem is to add residual diffusion to the solution by taking, $\eta_{D} \geqslant \eta_{A}$. The effect of this is to over-diffuse the solution to smooth cavitation oscillations, as in the CAFE/CASE method. By adding some anti-diffusion to the solution we hope to remove most of the effect of over diffusion on accuracy, but not its effect on oscillations. In taking this approach in applying the FE-FCT algorithm to cavitation problems we have found that the existing flux limiter Eq. (23) tends to over damp the solution even after the anti-diffusion stage. The explanation for this is that the strong flux limiter in Eq. (23) tends to over correct the anti-diffusive fluxes in some areas. In these regions strong flux limitation tends to remove the peak and replace it with a terraced region. This phenomenon is called clipping [5,41]. To overcome clipping and the over diffusive nature of strong flux limiting for $\eta_{D}>\eta_{A}$, we have adapted a one-sided flux limiter [4], given by Eq. (26), to Xiao's FE-FCT algorithm.

$$
\varphi_{i}^{c}=S \cdot \max \left\{0, \min \left(S \cdot \Delta_{i}^{\prime},\left|\varphi_{i}^{A}\right|\right)\right\}
$$

where, 


$$
\Delta_{i}^{\prime}=\dot{p}_{v, i+1}^{T D}-\dot{p}_{v, i}^{T D}
$$

Compared to strong flux limitation, one-sided flux limitation (Eq. (26)) allows more oscillations, however Eq. (26) gives higher accuracy in regions where the solution shows varies greatly from zero. (e.g. shock waves and cavitation regions) [5]. This is a key feature for cavitation problems because we treat cavitation oscillations by adding residual diffusion to the solution. Therefore, we require the anti-diffusion stage to be as accurate as possible in regions away from cavitation oscillations, where we have large amplitude peaks such as shock fronts and cavitation boundaries, to mitigate the effect of residual diffusion.

\section{Example and test problems}

\subsection{Shock wave in a fluid bar}

\subsubsection{Setup}

The first example problem is used to assess the shock wave capturing ability of the FE-FCT algorithms compared to the CAFE/CASE method. Details of the CAFE/CASE method used in this work are given in $[12,13,34,35]$. The problem, originally solved in [35] consists of a one-dimensional horizontal bar of acoustic fluid, with length $L=$ $6 \mathrm{~m}$. In acoustic fluid hydrostatic pressure is neglected and the fluid has properties $\rho_{o}=1025 \mathrm{~kg} / \mathrm{m}^{3}$ and $c=$ $1450 \mathrm{~m} / \mathrm{s}$. At the right end of the bar (taken as $x=L$ ) we apply a known pressure boundary given by a plane step exponential wave

$$
p(L, t)=P_{\text {peak }} \exp \left[-\frac{t}{\tau}\right],
$$

where, $P_{\text {peak }}$ is the peak pressure of the shock wave and $\tau$ is the decay constant. We take the peak pressure as $P_{\text {peak }}=16.12 \mathrm{MPa}$ and the decay constant as $\tau=0.423 \mathrm{~ms}$. Note that no cavitation occurs in this problem and that the left end boundary $(x=0)$ is neglected as the simulation is terminated before the shock wave reaches this point on the fluid bar.

For all CAFE and CASE simulations the damping parameter, $\beta$ [13], is selected as 0.50 . The CAFE/CASE damping parameter smoothes high frequency modes that can cause dispersive oscillations if left un-damped. For Xiao's FE-FCT algorithm we set the diffusion and anti-diffusion coefficients, $\eta_{D}=\eta_{A}=0.125$, and use the standard strong flux limiter (Eq. (23)). For the residual diffusion case we use the one-sided flux limiter (Eq. (26)) with diffusion and anti-diffusion coefficients, $\eta_{D}=0.125$ and $\eta_{A}=0.09$. All simulations use a time step of one half the CFL limit.

\subsubsection{Results}

Results are obtained using 81 degrees of freedom (DOF) and 241 DOF for each CAFE, CASE, and FE-FCT meshes. The CASE mesh uses 8th order elements and FE-FCT meshes use both Xiao's FE-FCT algorithm and our residual diffusion FE-FCT algorithm. Figure 1 shows the results for the 81 DOF case compared to the exact solution at times of $1 \mathrm{~ms}, 2 \mathrm{~ms}$, and $3 \mathrm{~ms}$. In terms of preserving the peak pressure of the shock wave, Fig. 1 shows Xiao's FE-FCT algorithm gives the best result. While the 8th order CASE solution and the residual diffusion FE-FCT algorithm give nearly identical results, both experience a significant loss in peak pressure as the wave propagates. The CAFE (1st order CASE) solution gives the poorest result, losing nearly half of the peak pressure magnitude $(16.12 \mathrm{MPa})$ by $t=3 \mathrm{~ms}$. Note that as mentioned in Section 2.2, the CAFE damping parameter sufficiently smoothes any spurious oscillations for the 8th order CASE solution. In terms of capturing the sharpness of the front, both CASE and Xiao's FE-FCT algorithm solutions have the sharpest wave fronts, with the CAFE solution having the most smeared wave front.

Increasing the DOF to 241, we see in Fig. 2 that the results of all methods are improved in both preserving peak pressure and capturing the sharpness of the wave front. Once again, Fig. 2 shows Xiao's FE-FCT algorithm to give the best result, propagating the shock wave with a small loss in peak pressure. The 8th order CASE and residual diffusion FE-FCT algorithm also give very similar results as in the 81 DOF case, but there is still a significant loss 


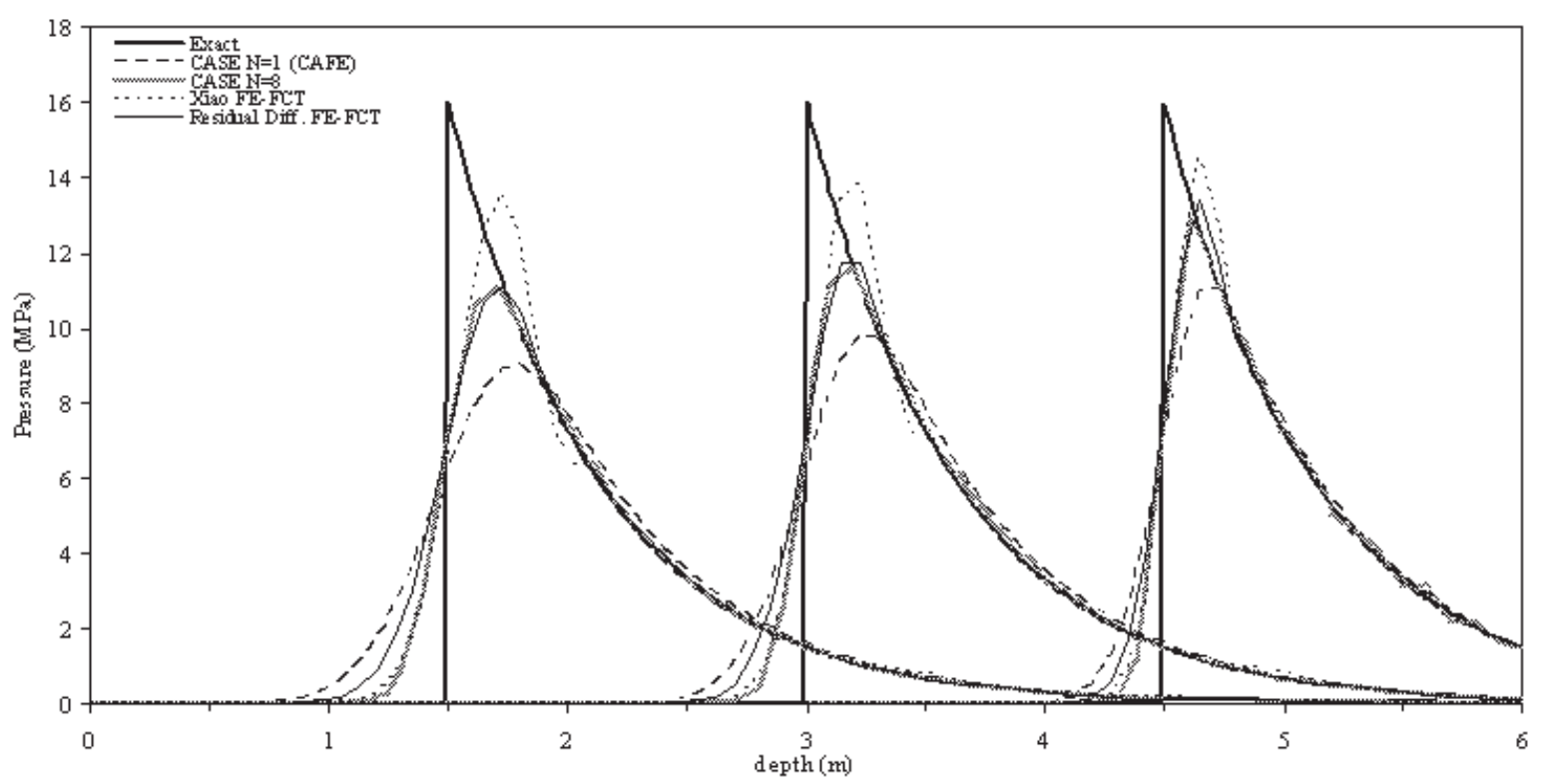

Fig. 1. Shock wave in a fluid bar results for 81 DOF mesh at times (from right to left) $t=1 \mathrm{~ms}, 2 \mathrm{~ms}$, and $3 \mathrm{~ms}$.

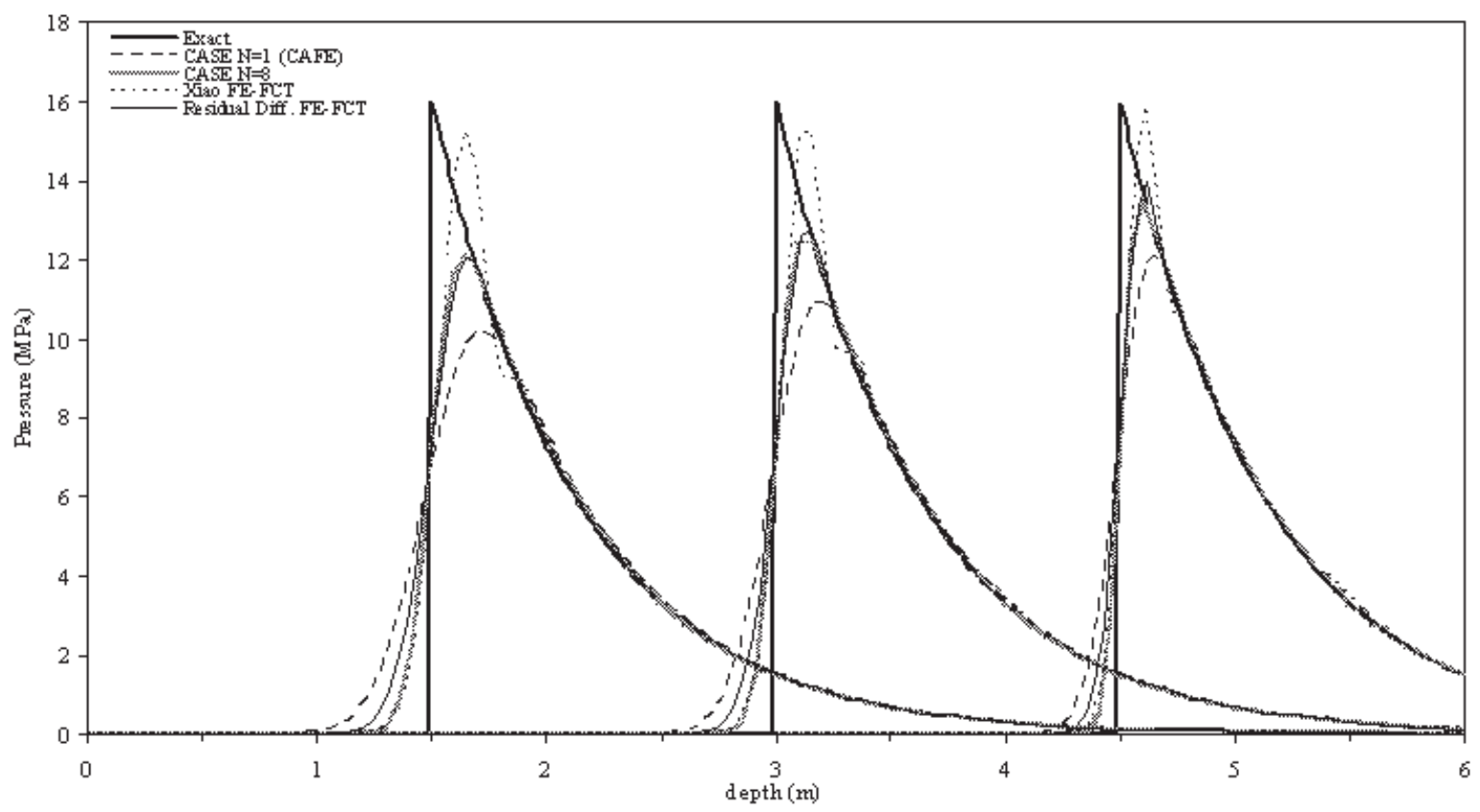

Fig. 2. Shock wave in a fluid bar results for $241 \mathrm{DOF}$ mesh at times (from right to left) $t=1 \mathrm{~ms}, 2 \mathrm{~ms}$, and $3 \mathrm{~ms}$.

of peak pressure. Also like the 81 DOF case both the 8th order CASE and Xiao's FE-FCT algorithm solutions have the sharpest wave fronts.

For each of the test cases presented, solutions obtained by Xiao's FE-FCT algorithm are, in terms of capturing the peak pressure of the shock wave, consistently more accurate than the CAFE, 8th order CASE, and residual diffusion 


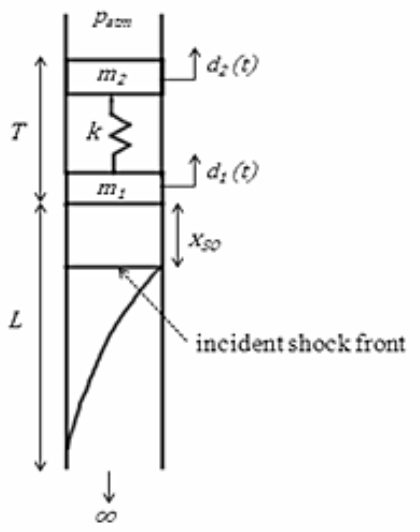

Fig. 3. Schematic of the mass-spring oscillator UNDEX problem.

FE-FCT algorithm. This is expected because for this example problem the solution can be sufficiently smoothed during the diffusion stage of the FE-FCT algorithm, allowing us to fully correct the solution in the anti-diffusion stage. Results using the residual diffusion FE-FCT algorithm were nearly identical to the 8th order CASE solution, but both methods experienced a large loss of peak pressure for even the most refined mesh. However, both the residual diffusion FE-FCT algorithm and CASE solutions were significantly better than the CAFE solutions. This shows that even without fully correcting the solution during the anti-diffusion stage, the modified limiter in the residual diffusion FE-FCT algorithm still allows improved accuracy over a standard linear finite element scheme without increasing spurious oscillations that arise from shock wave propagation. While this is an important result it does not consider on the effectiveness of the FE-FCT algorithm to reduce spurious oscillations that result from the formation of cavitation regions in the fluid.

\subsection{Two degree of freedom mass-spring oscillator}

\subsubsection{Setup}

To evaluate the effectiveness of the FE-FCT algorithm when applied to a far-field UNDEX problem with cavitation we use a two degree of freedom mass-spring oscillator problem shown in Fig. 3. The problem consists of a structure with masses $m_{1}$ and $m_{2}$, displacements $d_{1}(t)$ and $d_{2}(t)$, sitting on top of a column of fluid with depth $L$. The masses are connected by a spring with spring constant $k$ and are initially at rest. At time $t=0$ the wave front of the incident shock wave, modeled as a plane step exponential wave, is located at $x_{S O}$.

This problem was originally solved by Sprague and Geers in [33] using the CAFE approach and latter using the CASE approach [35]. Solutions using the approach of Nimmagadda and Cipolla are given in the ABAQUS manual [1] and most recently the problem has been solved in [40] using a ghost fluid method with a compressible flow solver. We solve this problem for $m_{2} / m_{1}=5$ in one-dimension using the CASE model in [35] and by applying both FE-FCT methods in one-dimension.

For this evaluation we wish to examine the effectiveness of the FE-FCT algorithms to both accurately capture cavitation phenomena and reduce cavitation oscillations. Therefore we use the scattered field model (SFM) of Sprague and Geers [34]. This allows comparison of CAFE, CASE, and FE-FCT in terms of capturing cavitation phenomena only, avoiding dispersive errors associated with propagating the incident shock wave through the unknown incident field in a total field model. Using SFM for our formulation requires the total pressure and pseudo pressure to be written as

$$
\begin{aligned}
p_{\tau} & =p_{I}+p_{s}+p_{h}, \\
p_{v, t} & =p_{v, I}+p_{v, s^{\prime}}
\end{aligned}
$$

where $p_{s}, p_{v, s}, p_{I}$, and $p_{v, I}$ are the scattered pressure, scattered pseudo pressure, incident pressure, and incident pseudo pressure respectively. The incident pressure (i.e. the shock wave) in this problem is given as [35] 


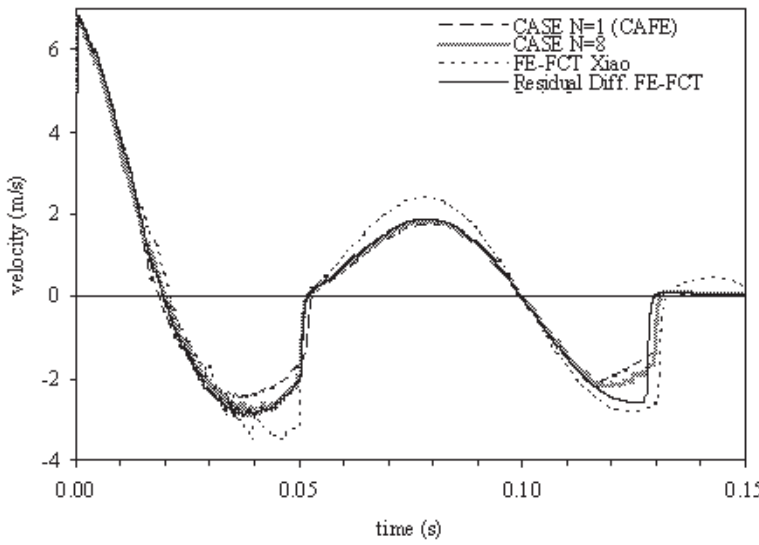

(a)

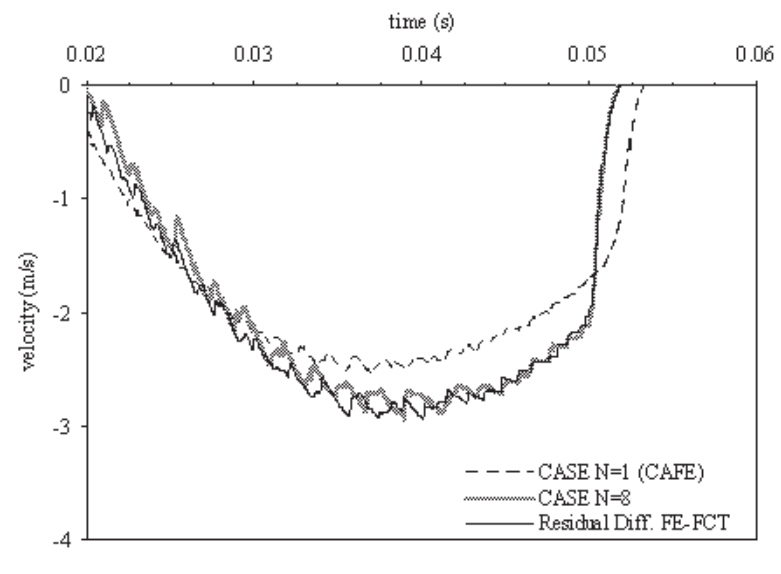

(b)

Fig. 4. Results for the velocity of $m_{1}$ on two time scales using 41 fluid DOF.

$$
p_{I}(x, t)=P_{\text {peak }} \exp \left[-\frac{t+\left(x-x_{S O}\right) / c}{\tau}\right] \mathcal{H}\left(t+\left(x-x_{S O}\right) / c\right),
$$

where $\mathcal{H}(t)$ is the Heaviside function, $P_{\text {peak }}$ is the peak pressure of the shock wave and $\tau$ is the decay constant. Also note that for SFM the fluid forcing term given by the boundary condition in Eq. (16) becomes (in one-dimension)

$$
\ddot{d}_{s, 1}=-\frac{1}{\rho_{o}} \frac{\partial p}{\partial x}
$$

where $\ddot{d}_{s, 1}$ is now the scattered acceleration of mass $m_{1}$. The masses are modeled as lumped mass elements and the infinite boundary treated with the Sommerfeld NRBC (Eq. (17)). The fluid and structure are coupled together using a staggered solution procedure similar to the one used in [13] for the CAFE method. The mass-spring system is integrated in time with the central difference method.

For the Sprague and Geers problem in [35] the fluid has depth $L=3.0 \mathrm{~m}$ and we take $\rho_{o}=1025 \mathrm{~kg} / \mathrm{m}^{3}, c=$ $1450 \mathrm{~m} / \mathrm{s}$, and $p_{\text {atm }}=101.3 \mathrm{kPa}$. The structure has a draft, $T=5.08 \mathrm{~m}$, a total mass, $m_{1}+m_{2}=5207 \mathrm{~kg}$, and $k=(10 \pi)^{2} m_{2}$. The peak pressure is $P_{\text {peak }}=16.12 \mathrm{MPa}$ the decay constant is $\tau=0.423 \mathrm{~ms}$ and $x_{S O}=0 \mathrm{~m}$. The incident shock wave used is equivalent to the shock wave generated by a $45.5 \mathrm{~kg} \mathrm{HBX}-1$ charge detonated $10.1 \mathrm{~m}$ below the fluid-structure interface [35].

For both CAFE and CASE solutions the damping parameter, $\beta$ [13], is set to 0.50. As in the first example (Section 5.1) we set the diffusion and anti-diffusion coefficients, $\eta_{D}=\eta_{A}=0.125$, and use the standard strong flux limiter (Eq. (23)) for Xiao's FE-FCT algorithm. For the residual diffusion case with the one-sided flux limiter (Eq. (26)) we use diffusion and anti-diffusion coefficients, $\eta_{D}=0.125$ and $\eta_{A}=0.09$. All results are obtained using an in-house FORTRAN 95 code. For all methods studied the simulation is run to a termination time of $0.15 \mathrm{~s}$ and the time step is set at half the CFL limit.

\subsubsection{Results - velocity response}

We begin by comparing the CAFE method and the 8th order CASE method to both FE-FCT algorithms using a uniform meshes consisting of $41 \mathrm{DOF}, 81 \mathrm{DOF}$, and $161 \mathrm{DOF}$. Results for the vertical velocity of $m_{1}$ for each mesh are given in Figs 4, 5, and 6.

For each method $m_{1}$ responds to the incident wave with a sharp rise in velocity to a peak or "kick-off" velocity of $\sim 6.5 \mathrm{~m} / \mathrm{s}$. After this velocity is reached, the velocity begins to decay and take on a negative value as the cavitation region in the fluid grows. At $0.05 \mathrm{~s}$ the pressure wave generated by the closure of the cavitation region causes a second sharp rise in velocity. Note in our discussion of the results we will refer to this time as the re-load time. The 


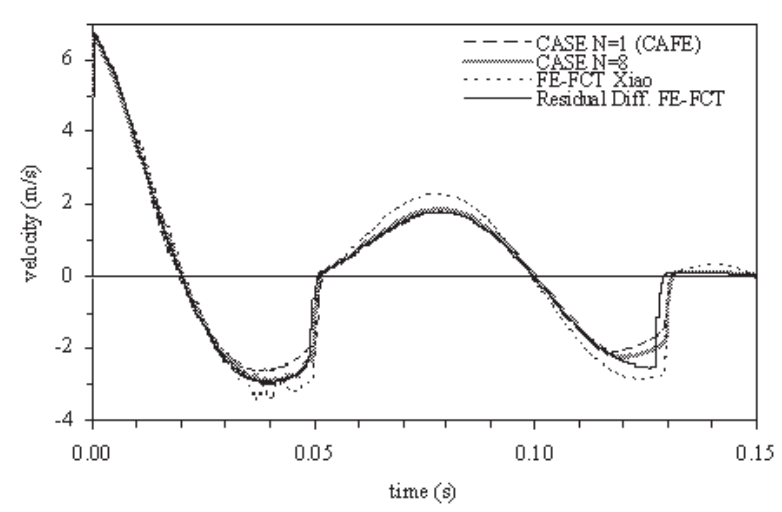

(a)

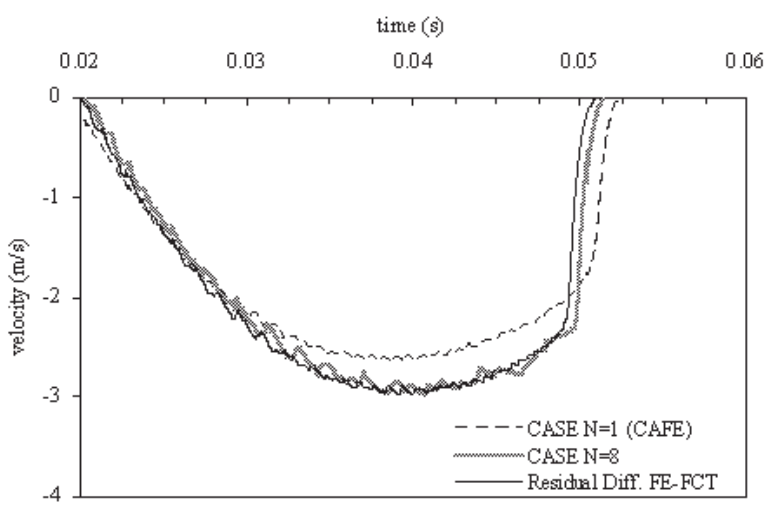

(b)

Fig. 5. Results for the velocity of $m_{1}$ on two time scales using 81 fluid DOF.

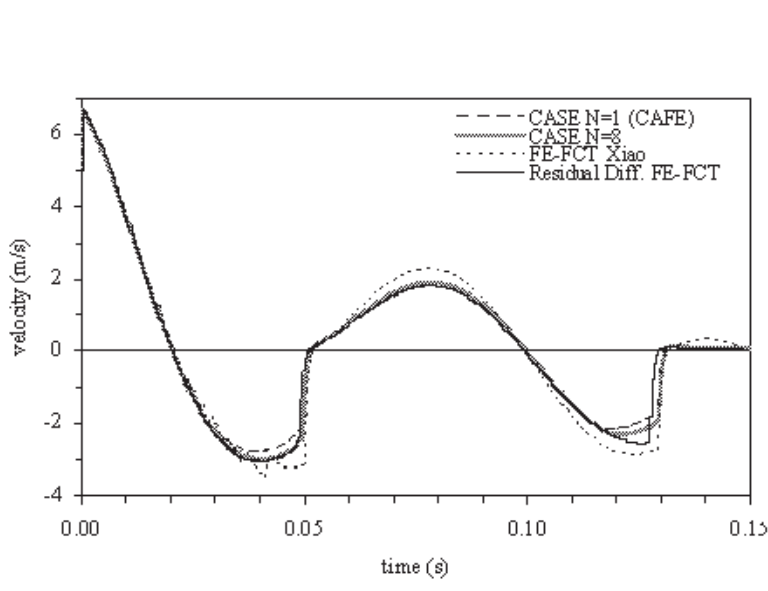

(a)

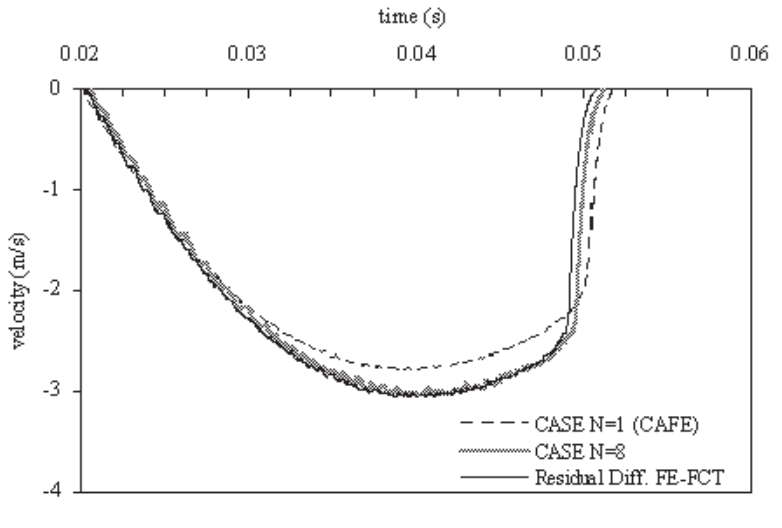

(b)

Fig. 6. Results for the velocity of $m_{1}$ on two time scales using 161 fluid DOF.

upward motion of the mass system resulting from the closure pulse causes a secondary cavitation region to form. After a rise to a second peak velocity the mass once again begins to fall into the fluid until the arrival of the closure pulse from the second cavitation region causes a sharp velocity increase at $0.13 \mathrm{~s}$. The second cavitation region is not as large as the first, occurring mainly in the fluid directly beneath the structure [34].

Figure 4 a gives the $m_{1}$ velocity response using a 41 DOF mesh for each method. A closer view of the velocity response during the first cavitation region is given in Fig. 4b. Figure 5b shows that $m_{1}$ is most susceptible to spurious pressure oscillations between $0.02 \mathrm{~s}$ and $0.05 \mathrm{~s}$. Comparing the methods in Fig. $4 \mathrm{~b}$ it is seen that the CAFE solution does not exhibit any meaningful spurious oscillations and does not match the 8th order CASE solution. Xiao's FE-FCT method has the largest negative velocity magnitude during this time frame which corresponds to the prediction of a larger cavitation region in the fluid. However, Xiao's FE-FCT method suffers from large spurious pressure oscillations which degrade the quality of the solution. In these oscillations we see the effects of the problems associated with applying the Xiao's FE-FCT scheme to the far-field UNDEX problem, the most significant of which is that the flux limiter cannot remove spurious oscillations unless the solution can be adequately smoothed during 


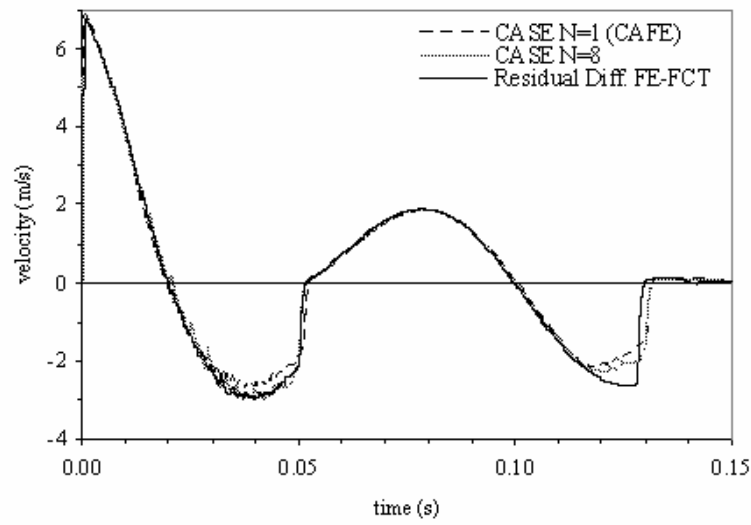

(a)

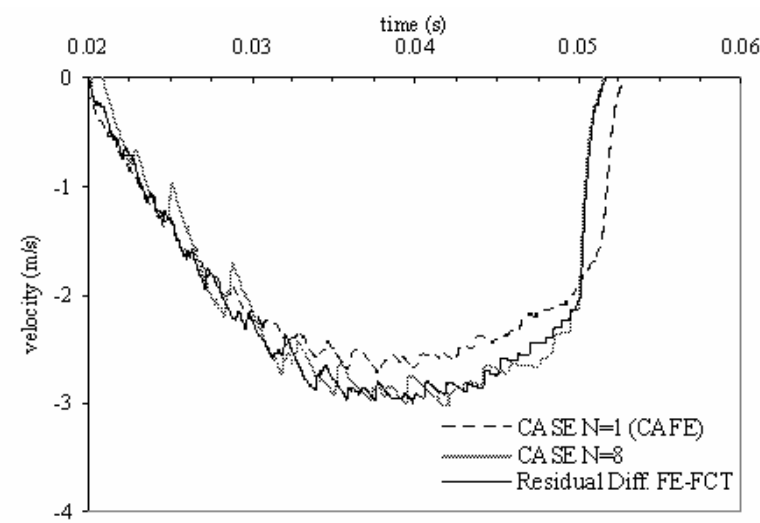

(b)

Fig. 7. Comparison of the $\mathrm{m} 1$ velocity response on two time scales using 41 DOF with $\beta=0.25$ for CAFE/CASE and $\eta_{D}=0.125$ and $\eta_{A}=$ 0.10 for residual diffusion FE-FCT.

the diffusion stage. During the first cavitation region response period $(0.02 \mathrm{~s}-0.05 \mathrm{~s})$, Fig. $4 \mathrm{~b}$ shows the residual diffusion FE-FCT scheme is nearly identical to the 8th order CASE solution. Note the residual diffusion FE-FCT scheme does not suffer from the severe oscillations that the original FE-FCT method does. Figure $4 \mathrm{~b}$ also shows a small reduction in spurious oscillations when compared to the CASE solution, especially during the $0.02 \mathrm{~s}-0.03 \mathrm{~s}$ timeframe.

Figures 5 and 6 gives $m_{1}$ velocity response results for each method using an 81 DOF and 161 DOF mesh respectively. As in the previous results, Figs 5a and 6a show that Xiao's FE-FCT algorithm gives the largest negative velocity magnitude during the $0.02 \mathrm{~s}-0.05 \mathrm{~s}$ timeframe, but again suffers from large spurious oscillations. Note that unlike the CASE/CAFE and residual diffusion FE-FCT results, refining the mesh does not have a large influence on the spurious oscillations in the results obtained with Xiao's FE-FCT algorithm. Again the CAFE solution does not exhibit any oscillations in the velocity response, especially for the 161 DOF results in Fig. 6, but does not agree well with the 8th order CASE solution.

Figures $5 \mathrm{~b}$ and $6 \mathrm{~b}$ show that the 8th order CASE and residual diffusion FE-FCT results agree well during the response to the first cavitation region. In addition, as in the 41 DOF results, Figs $5 \mathrm{~b}$ and $6 \mathrm{~b}$ also show that the residual diffusion FE-FCT algorithm again reduces the larger 8th order CASE oscillations, especially in the 81 DOF results. However we note that for all models the refinement of the mesh from 41 DOF to 161 DOF significantly reduces oscillations in the velocity response.

However, comparing the $41 \mathrm{DOF}, 81 \mathrm{DOF}$, and $161 \mathrm{DOF}$ results during the time period that the second cavitation region occurs $(0.05 \mathrm{~s}-0.13 \mathrm{~s})$, both FE-FCT methods exhibit different results than the CAFE and CASE results. Both FE-FCT results show a larger magnitude of negative velocity, a sharper rise in positive velocity magnitude, and an earlier closure time in response to the second cavitation region. This result is unexpected as both FE-FCT algorithms showed good agreement with the CASE/CAFE results during the shock and first cavitation region responses. This difference occurs because during the collapse of the second cavitation region the FE-FCT models do not predict the small region of fluid accretion (a region of un-caviated fluid between the structure and upper cavitation boundary [33]) that develops in the CAFE/CASE models. Because the layer of fluid accretion, which slows the velocity response during the collapse of the second cavitation region in the CAFE/CASE results, does not develop in the FE-FCT models a larger negative velocity magnitude and a sharper rise in positive velocity are observed. Despite this difference, we note that Figs 4-6 show that the difference between the CAFE/CASE and FE-FCT results during the response to the second cavitation region lessens as the mesh is refined from $41 \mathrm{DOF}$ to $161 \mathrm{DOF}$.

The comparisons in Figs 4-6 are based on unique choices of numerical parameters for both the CAFE/CASE results and the FE-FCT results. Thus in Figs 7 and 8 we compare CAFE, CASE, and residual diffusion FE-FCT on 


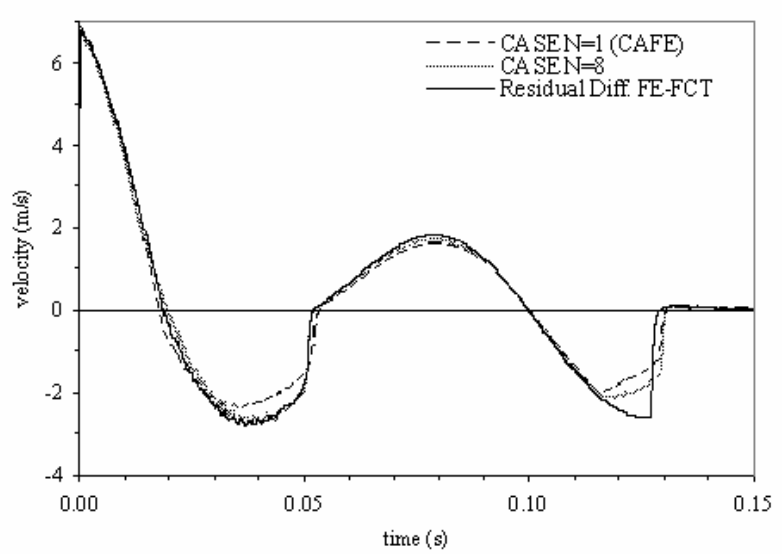

(a)

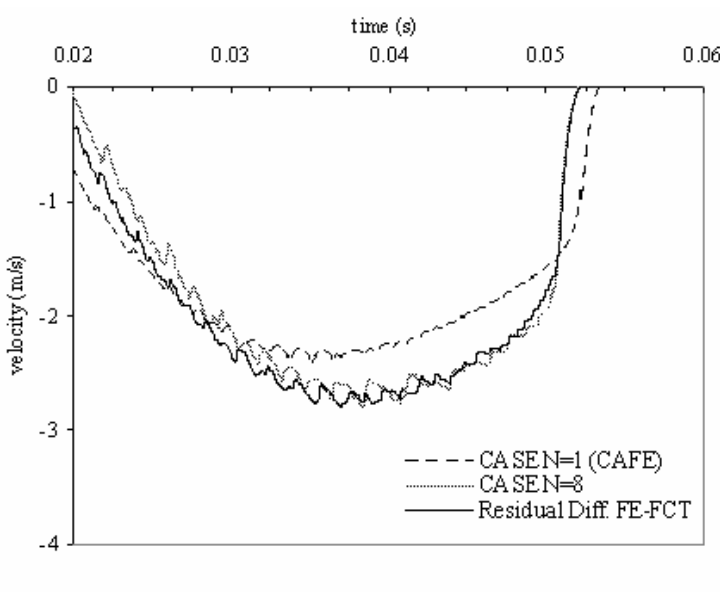

(b)

Fig. 8. Comparison of the $\mathrm{m} 1$ velocity response on two time scales using 41 DOF with $\beta=1.00$ for CAFE/CASE and $\eta_{D}=0.125$ and $\eta_{A}=$ 0.07 for residual diffusion FE-FCT.

a 41 DOF mesh using different values of $\beta, \eta_{D}$, and, $\eta_{A}$. Note that results using Xiao's FE-FCT algorithm are not included due to the fact $\eta_{D}=\eta_{A}=0.125$ is given as the optimal choice for the algorithm and because the results obtained with the algorithm produced unacceptable oscillations in the velocity response.

Figure 7 gives the velocity response results using $\beta=0.25$, which is the smallest value recommended in [34], for the CAFE and 8th order CASE models and $\eta_{D}=0.125$ and $\eta_{A}=0.10$ for the residual diffusion FE-FCT algorithm. Figure 8 shows that the reduction in $\beta$ and the increase in $\eta_{A}$ increases oscillations in the velocity response but also increases the magnitude of negative velocity reached during the response to the cavitation regions for each model when compared to the $\beta=0.50$ and $\eta_{D}=0.125$ and $\eta_{A}=0.09$ cases given in Fig. 4 . As found previously, an examination of the response to the first cavitation region in Fig. 7a shows that the residual diffusion FE-FCT results agree well with the 8th order CASE results and reduce the larger oscillations observed in the CASE velocity response. The reduction in oscillations for the $41 \mathrm{DOF}$ case with $\beta=0.25$ and $\eta_{D}=0.125$ and $\eta_{A}=0.10$ appears to be more significant than that observed previously in Fig. $5 \mathrm{~b}$ (with $\beta=0.50$ and $\eta_{D}=0.125$ and $\eta_{A}=0.09$ ). Note that the differences observed between CAFE/CASE and the FE-FCT results during the response to the second cavitation region still occur with the change in numerical parameters.

Figure 8 gives the velocity response results using $\beta=1.00$, which was used in [12,13,34], for the CAFE and 8th order CASE models and $\eta_{D}=0.125$ and $\eta_{A}=0.07$ for the residual diffusion FE-FCT algorithm. The increase in $\beta$ and the decrease in $\eta_{A}$ cause a reduction in both oscillations and negative velocity magnitudes reached during the response to cavitation. This is particularly evident for the CAFE results, which show a significant change in velocity response when compared to the $\beta=0.50$ case in Fig. 4. Figure $8 \mathrm{~b}$ shows that even with the large amount of damping applied to the model, the 8th order CASE results still exhibit visible oscillations in the velocity response to the first cavitation region. Figure $8 \mathrm{~b}$ also shows that the residual diffusion FE-FCT results again agree well with the 8th order CASE results and again succeeds in reducing some of the larger CASE oscillations. Again we note that the differences observed between CAFE/CASE and the FE-FCT results during the response to the second cavitation region again occur despite the change in numerical parameters.

Based on these results, Xiao's FE-FCT algorithm with strong flux limiting is not effective for problems with cavitation because of the large oscillations that occur in the structural response. The residual diffusion FE-FCT algorithm offers solutions with an accuracy level comparable to that of an 8th order CASE solution for the duration of the first cavitation region. The residual diffusion FE-FCT algorithm also succeeds in reducing some of the spurious oscillations associated with CASE solutions. However, there is a significant difference between the CAFE/CASE results and the residual diffusion FE-FCT results during the response to the second cavitation region. We investigate this difference in results further in Section 5.3 by comparing CAFE, CASE, and the residual diffusion FE-FCT 


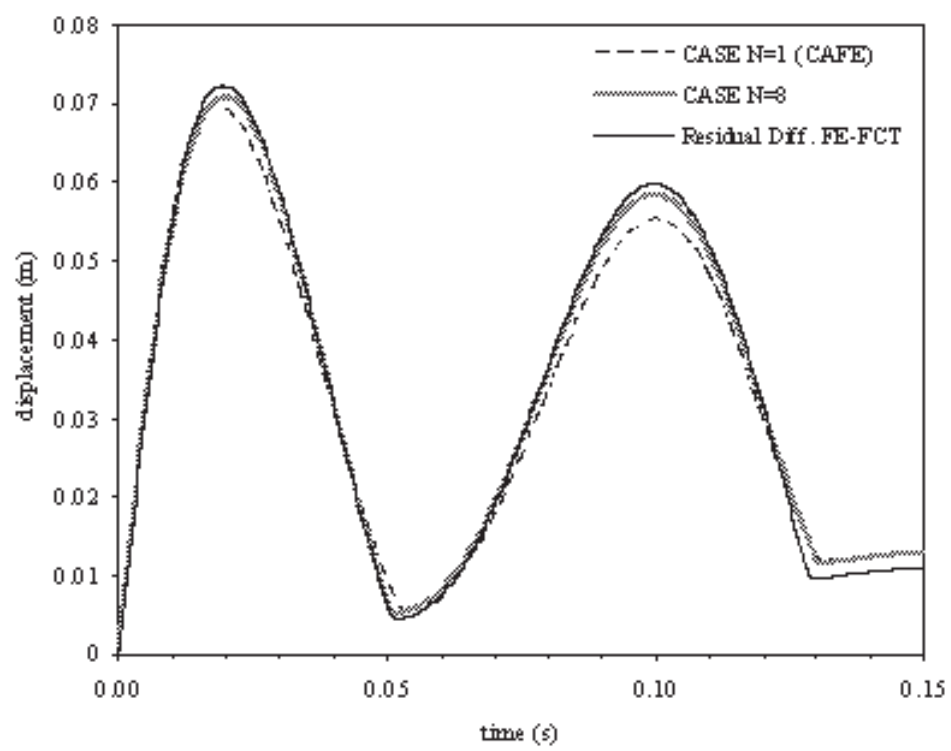

Fig. 9. Results for the displacement of $m_{1}$ using 41 fluid DOF.

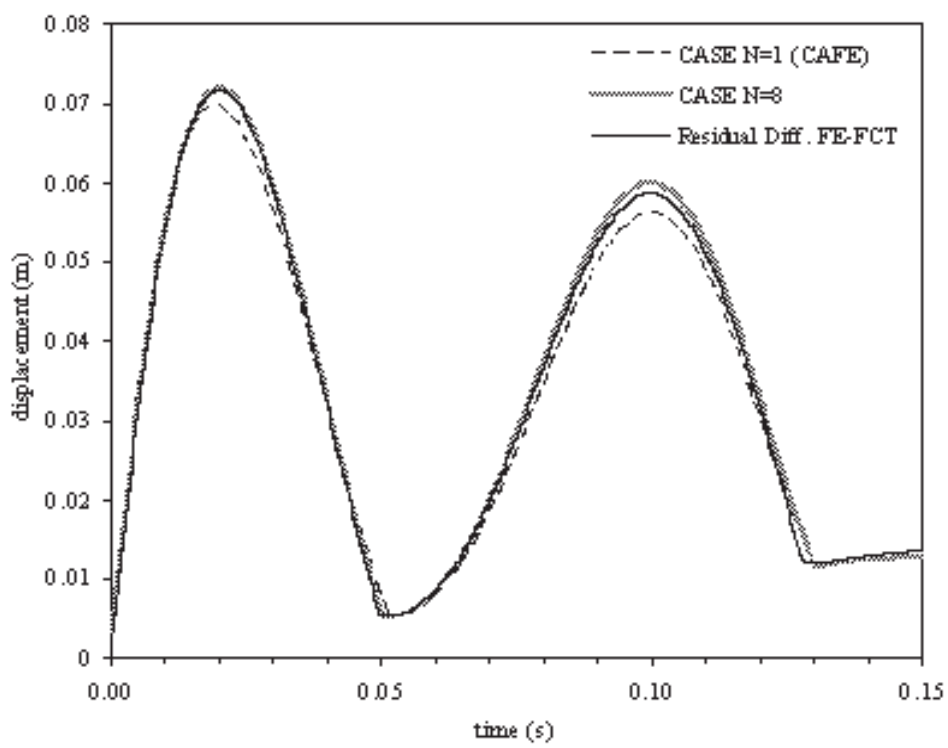

Fig. 10. Results for the displacement of $m_{1}$ using 81 fluid DOF.

algorithm for the Bleich-Sandler problem [3] which has the only known benchmark solution for this type of cavitation problem.

\subsubsection{Results - displacement response}

Previous analysis of the two degree-of-freedom mass spring oscillator problem in $[1,33,34,40]$ has focused on the velocity response of the masses. To understand the structural response more completely we present results for the displacement response of $m_{1}$ using the $41 \mathrm{DOF}, 81 \mathrm{DOF}$, and 161 DOF fluid models in Figs 10-12 respectively. 


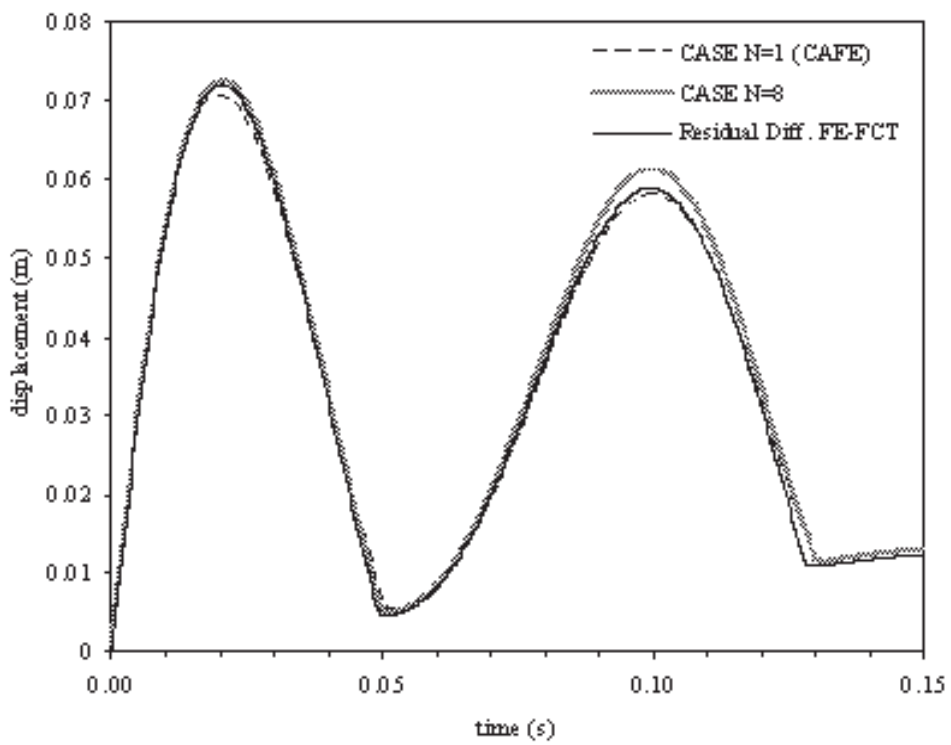

Fig. 11. Results for the displacement of $m_{1}$ using 161 fluid DOF.

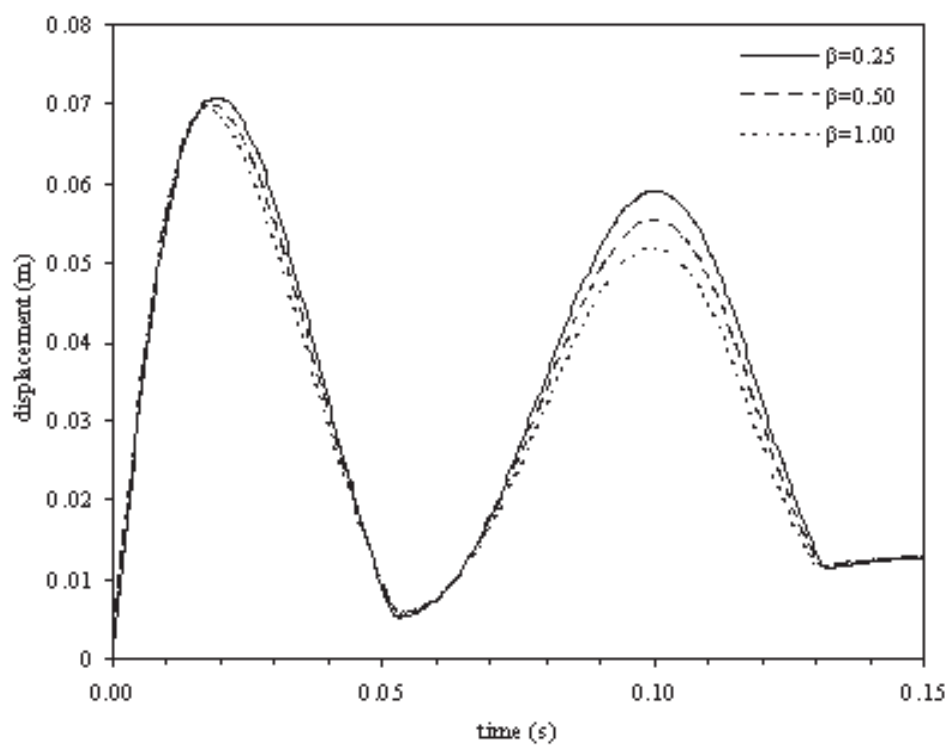

Fig. 12. Comparison of the displacement of $m_{1}$ using a 41 DOF CAFE mesh with, $\beta=0.25, \beta=0.50$, and $\beta=1.00$.

Interestingly, none of the results in Figs 9-11 exhibit any of the oscillations visible in the velocity response results. Comparing CAFE, 8th order CASE, and the residual diffusion FE-FCT results for each level of mesh refinement there is a small difference in the maximum displacements reached during the response to the first cavitation region. This difference is primarily observed between the CAFE results and the 8th order CASE and residual diffusion FE-FCT results, with the CASE and FE-FCT results generally agreeing well during this period of the response.

A much larger difference in the maximum displacement during the response to the second cavitation region is also observed. This is especially true when comparing the 8th order CASE and CAFE result during this portion 


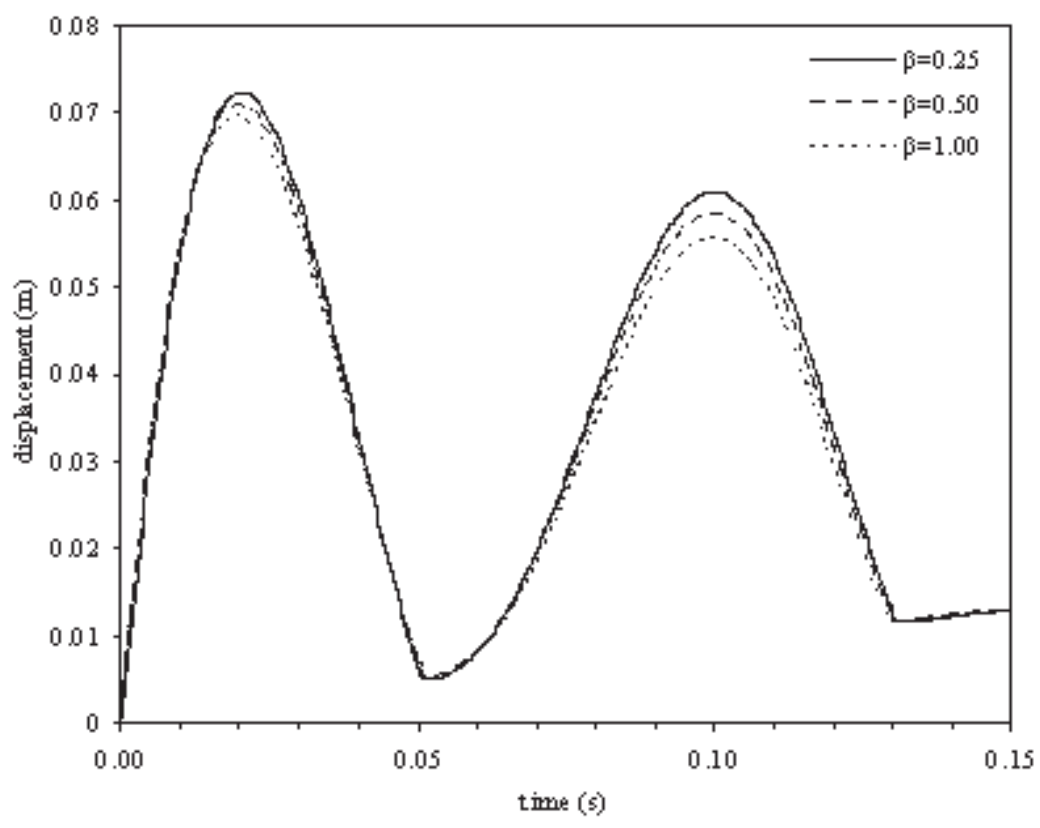

Fig. 13. Comparison of the displacement of $m_{1}$ using a 41 DOF 8 th order CASE mesh with, $\beta=0.25, \beta=0.50$, and $\beta=1.00$.

of the response. Here, there is an observable difference between the CASE and FE-FCT results not seen in the displacement response to the first cavitation region. Note that as in the early closure time of the second cavitation region in the FE-FCT velocity response results is also visible in the displacement response results around $t=0.125 \mathrm{~s}$ in Figs 9-11.

Figures 12, 13, and 14 give results for the 41 DOF CAFE, 8th order CASE, and residual diffusion FE-FCT models using the different values of $\beta, \eta_{D}$, and, $\eta_{A}$ studied in Section 5.2.2 for the velocity response. For the CAFE results in Fig. 12 we observe a small difference in the maximum displacement during the first cavitation region when comparing the $\beta=0.25$ case to the $\beta=1.00$ case, with the increased $\beta$ case giving a smaller displacement. A larger difference between the maximum displacement during the response to the second cavitation region for each value of $\beta$ is also observed. Again, the larger values of $\beta$ give smaller maximum displacements. Similar results are observed in Fig. 13 for the 8th order CASE results, although the change in $\beta$ causes a more visible difference in the maximum displacement reached during the response to the first cavitation region for the CASE model than in the CAFE model. For the residual diffusion FE-FCT results in Fig. 14 the difference in causes very little difference for the maximum displacement during the first cavitation region, but similar to the CAFE and CASE results the decrease in causes a smaller maximum displacement during the second cavitation region.

The differences in the maximum displacements during the response to cavitation occur because the maximum displacement is determined by the values of positive velocity magnitude reached during the response to cavitation. For example, during the response to the first cavitation region, the maximum displacement is determined by decay rate of positive velocity magnitude from the peak velocity to zero velocity [3]. This is in contrast to the case where no cavitation occurs and the displacement of the mass is proportional to the impulse of the incident wave [9]. In the non-cavitating fluid problem the impulse of the fluid loading of the structure is considered to be conserved despite the addition of artificial damping [24] or refinement of the fluid mesh. From the velocity response results in Figs. 4-8 we have found the velocity response is directly affected by, the fluid model itself (i.e. CAFE, CASE, or FE-FCT), the choice of numerical parameters in the fluid model (i.e. $\beta, \eta_{D}$, and, $\eta_{A}$ ), and the level of refinement in the fluid mesh. Thus, the differences observed in the displacement results (Figs 9-14) for each fluid model indicate errors in the capture of cavitation in the fluid model have an effect on the structural response as well. This indicates impulse may not drive the structural response to cavitation regions as it does when cavitation does not occur in the problem. 


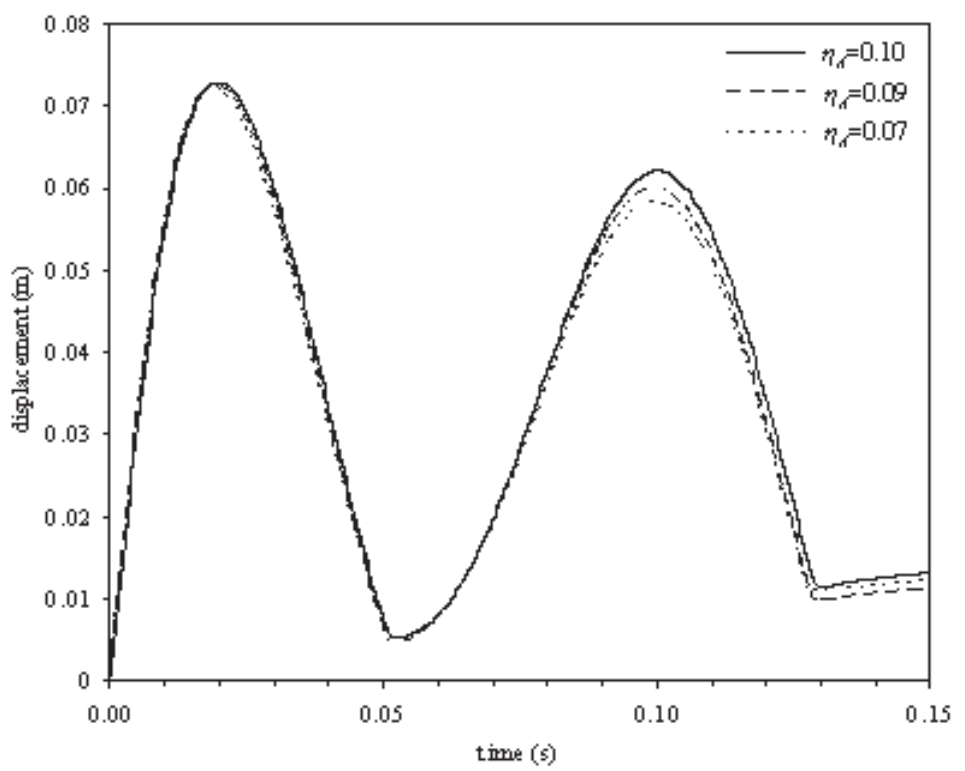

Fig. 14. Comparison of the displacement of $m_{1}$ using a 41 DOF residual diffusion FE-FCT mesh with, $\eta_{A}=0.10, \eta_{A}=0.09$, and $\eta_{A}=0.07$ (note all cases use $\eta_{D}=0.125$ ).

\subsection{Bleich-Sandler problem}

\subsubsection{Setup}

Because of the differences in response to the second cavitation region observed between the CAFE/CASE and FE-FCT results in this section we compare these methods to the only available benchmark problem. This benchmark problem is the Bleich-Sandler problem presented in [3]. The Bleich-Sandler problem consists of a mass sitting at the free surface of an infinite fluid column. The mass is subjected to a shock load, in the form of a step exponential wave, which causes cavitation in the fluid after reflection from the mass. A solution of this problem via the method of characteristics is given by Bleich-Sandler in [3]. Using the mass-spring oscillator structural model given in Section 5.2.1, we set $m_{1}=144 \mathrm{~kg}, m_{2}=0, k=0$, and take the draft of the mass as, $T=0.141 \mathrm{~m}$. Following [12, $13,33]$ the fluid depth $L=3.81 \mathrm{~m}$ and we take $c=1450 \mathrm{~m} / \mathrm{s}$ and $\rho_{o}=998 \mathrm{~kg} / \mathrm{m}^{3}$.

Again we use the scattered field model for both CAFE/CASE and the residual damping FE-FCT fluid models. Thus the known incident field is found using Eq. (30). At time $t=0$ the front of the incident shock wave is located at $x_{S 0}=0 \mathrm{~m}$, with peak pressure is $P_{\text {peak }}=0.712 \mathrm{MPa}$ and decay constant $\tau=0.999 \mathrm{~ms}$. Again for all solutions the time step is set at half the CFL limit.

\subsubsection{Results}

Figure 15 gives the results for the vertical velocity of $m_{1}$ for a 101 DOF CAFE solution, a 97 DOF 8th order CASE solution, and a 101 DOF residual diffusion FE-FCT solution. In Fig. 16, the CAFE/CASE results are generated using $\beta=0.50$ and the FE-FCT results are generated using $\eta_{D}=0.125$ and $\eta_{A}=0.09$. The 8 th order CASE and residual diffusion FE-FCT results agree very well, and capture the velocity magnitudes between $t=0.010 \mathrm{~s}$ and $t=0.012 \mathrm{~s}$ better than the CAFE model. As in the previous section, the FE-FCT model succeeds in reducing some of the larger oscillations that are observed in the 8th order CASE results. Furthermore, we do not observe any difference in the response to the cavitation region between the CAFE/CASE and FE-FCT results as we did in during the response to the second cavitation region in the two-degree of freedom mass spring oscillator problem. This is expected because the difference only occurred for the small secondary region of cavitation that does not develop in the Bleich-Sandler problem. 


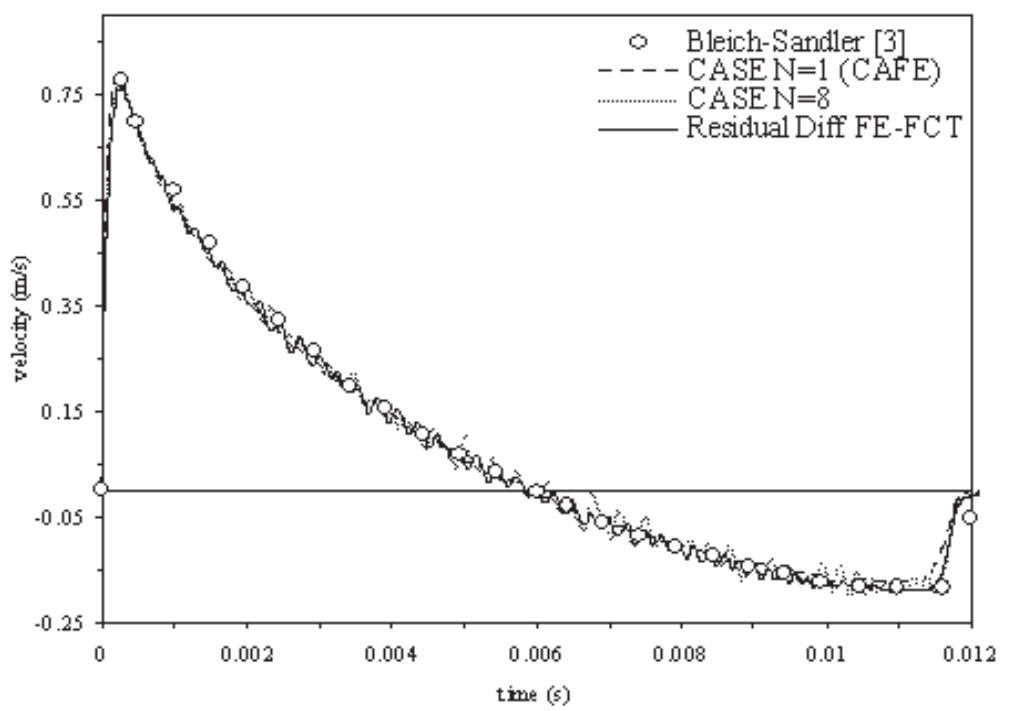

Fig. 15. Bleich-Sandler problem results for the velocity of $m_{1}$ using CAFE $(\beta=0.50)$, 8 th order CASE $(\beta=0.50)$, and residual diffusion FE-FCT $\left(\eta_{D}=0.125, \eta_{A}=0.09\right)$

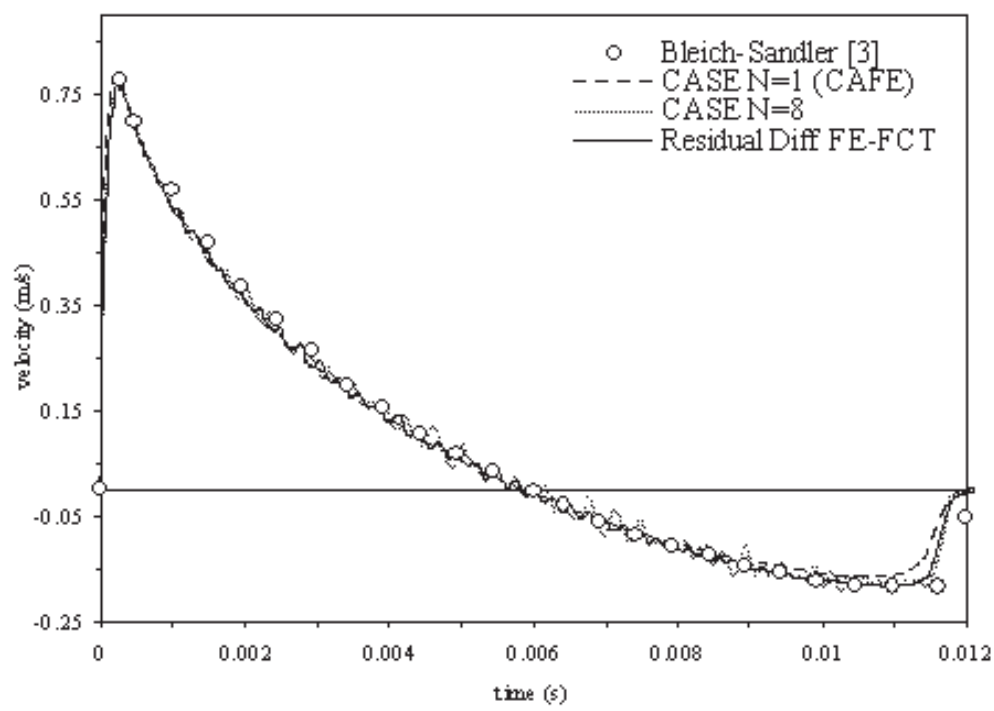

Fig. 16. Bleich-Sandler problem results for the velocity of $m_{1}$ using CAFE $(\beta=1.00)$, 8 th order CASE $(\beta=1.00)$, and residual diffusion FE-FCT $\left(\eta_{D}=0.125, \eta_{A}=0.07\right)$

As in the previous section and in $[12,13]$ we also wish to determine the effect of the numerical parameters $\beta$, $\eta_{D}$, and, $\eta_{A}$ on the structural response. Figure 16 shows results generated with $\beta=1.00$ and the FE-FCT results are generated using $\eta_{D}=0.125$ and $\eta_{A}=0.07$. Again we find the increase in $\beta$ and the decrease in $\eta_{A}$ cause an decrease in oscillations. However, we note that the oscillations in the 8th order CASE and FE-FCT results are still substantially larger than the CAFE results which exhibit no visible oscillations. Reducing $\beta$ to 0.25 and increasing $\eta_{A}$ to 0.10 , in Fig. 17, we find oscillations, especially in the 8th order CASE results, are increased from the $\beta=$ 0.50 and $\eta_{A}=0.09$ case. It is interesting to note the reduction of $\beta$ in the CAFE model gives a result that is almost 


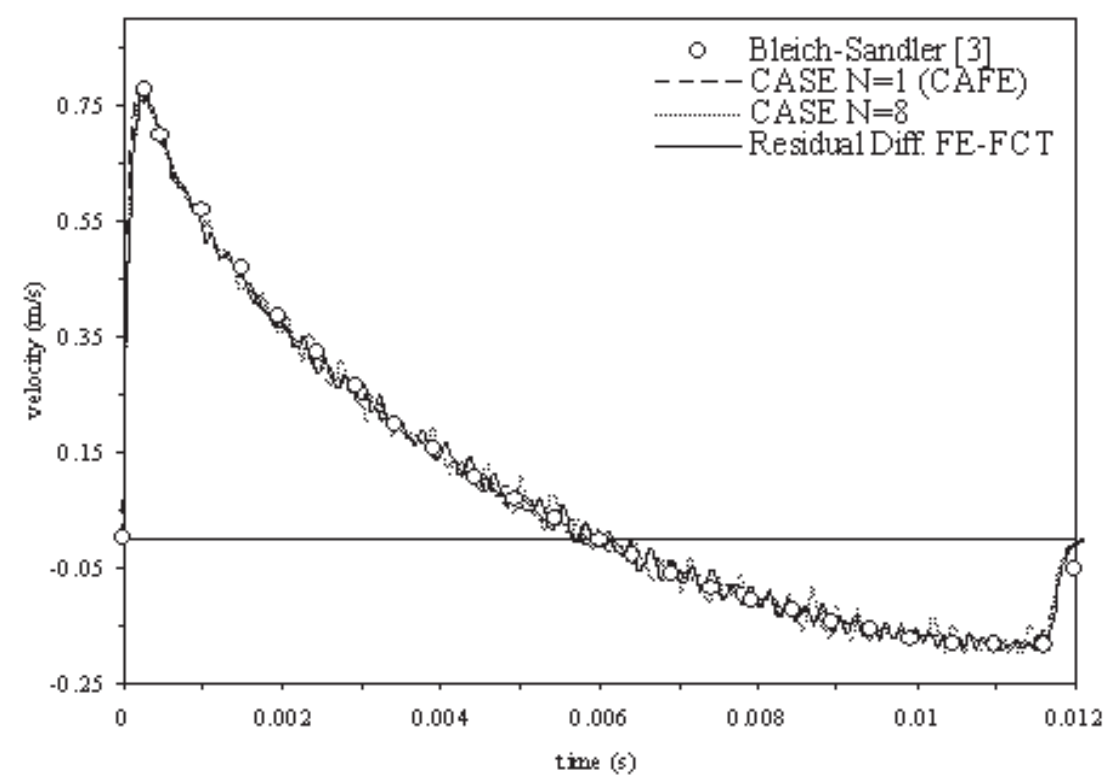

Fig. 17. Bleich-Sandler problem results for the velocity of $m_{1}$ using CAFE $(\beta=0.25)$, 8 th order CASE $(\beta=0.25)$, and residual diffusion $\operatorname{FE}-\mathrm{FCT}\left(\eta_{D}=0.125, \eta_{A}=0.10\right)$.

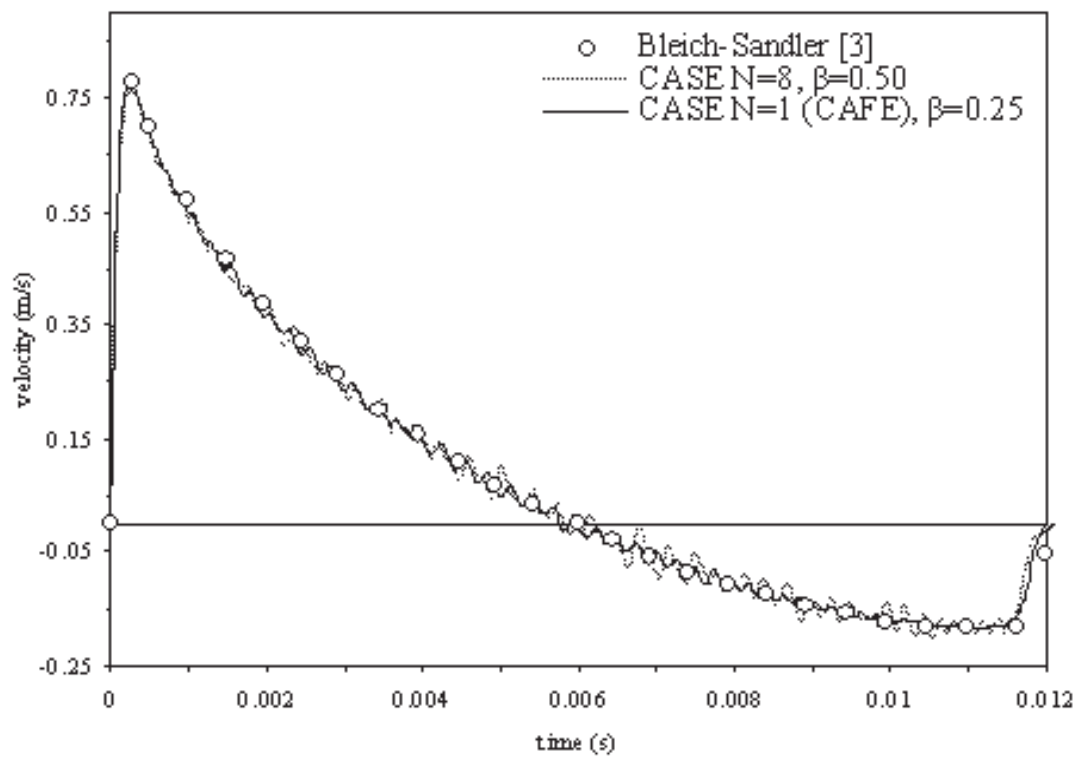

Fig. 18. Comparison of the velocity of $m_{1}$ for the Bleich-Sandler problem using CAFE with $\beta=0.25$ and 8 th order CASE with $\beta=0.50$.

identical to the 8th order CASE and FE-FCT results. This is demonstrated in Fig. 18, which compares the $\beta=0.50$ 8th order CASE result to the $\beta=0.25$ CAFE result. From this result we see that in the Bleich-Sandler problem it is possible to obtain results with a CAFE model that match higher order CASE results and do not exhibit as many oscillations simply by reducing the value of $\beta$ in the CAFE model. 


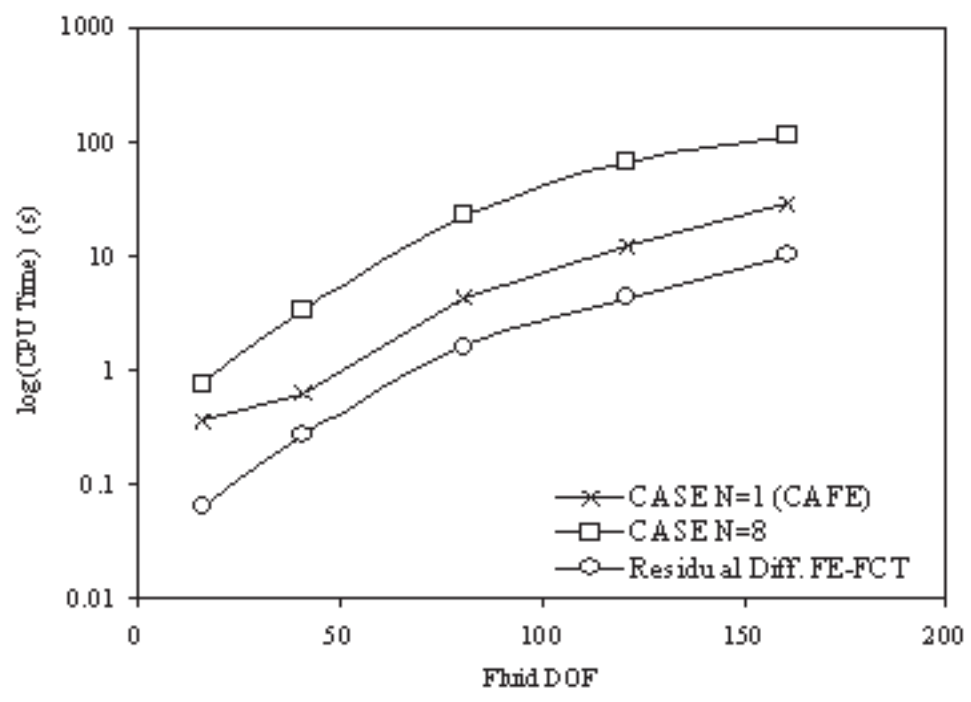

Fig. 19. Comparison of the CPU time required to solve the mass-spring oscillator problem for a given number of DOF for the CAFE ( $\beta=0.50$ ), CASE $(\beta=0.50)$, and FE-FCT $\left(\eta_{D}=0.125, \eta_{A}=0.09\right)$ models.

\subsection{Comments on computational efficiency}

In Sections 5.2 and 5.3 we demonstrated for two cavitation problems that the results of a high order CASE model could be matched, with fewer oscillations in the structural response, by a CAFE model. For the two-degree of freedom mass-spring oscillator problem this is best accomplished using the residual diffusion FE-FCT algorithm and in the Bleich-Sandler problem this is best accomplished by reducing the amount of artificial damping applied to the CAFE model. As discussed in Section 2.2, spectral elements offer a computational savings over linear FE only because the required DOF for an adequate solution is less than that of a linear FE solution. Thus, by obtaining a solution using the same DOF in the fluid model that is similar to the CASE solution using linear finite elements with FE-FCT or CAFE, the computational savings can be increased even more. We demonstrate the potential for increased computational savings in Fig. 19, which shows a comparison of CPU time required to solve the mass-spring oscillator problem in Section 5.2 for a number of fluid DOF. The CAFE/CASE results in Fig. 19 use $\beta=0.50$ and the FE-FCT results are generated use $\eta_{D}=0.125$ and $\eta_{A}=0.09$.

Note that in Fig. 19 the CAFE/CASE and FE-FCT algorithms solve different sets of equations and the addition of the FCT algorithm will make the FE-FCT naturally more computationally expensive when compared to an un-damped CAFE model. However, because both CAFE and CAFE require damping in cavitation problems, comparison of the residual diffusion FE-FCT algorithm to the 8th order CASE model in Fig. 19 shows that the FE-FCT algorithm gives a significant reduction in computational time even with the additional expensive of the FCT algorithm. Figure 19 also demonstrates that, for the same fluid DOF, the CAFE model gives a reduction in computational time over the 8th order CASE model.

\section{Conclusions}

Past work [34-36] has concluded that the capture of cavitation phenomena in numerical models of far-field UNDEX can be improved by applying the CASE methodology at the cost of increasing spurious oscillations in the problem domain. The results of this work showed that these oscillations affect the velocity response of the structure and are not well smoothed by the artificial damping scheme in CASE. A study of the displacement response in the two-degree of freedom mass-spring oscillator problem reveled the capture of the maximum structural displacements 
in response to the formation cavitation regions is dependent on the type of fluid model, the refinement of the fluid model, and the amount of artificial damping applied to the fluid model. Comparing CAFE, CASE, and the FE-FCT algorithm developed by Xiao, in the two-degree of freedom mass-spring oscillator problem, showed the FE-FCT algorithm, which uses linear finite elements on structured meshes, used with residual diffusion and a one-sided flux limiter, is effective in reducing the larger oscillations associated with the CASE method while maintaining the increased accuracy. We also found application of the FE-FCT algorithm caused a difference in the capture of fluid accretion in small secondary regions of cavitation. Comparison of CAFE, CASE, and the FE-FCT algorithm for the Bleich-Sandler problem showed no difference in cavitation capture between the CASE and FE-FCT models and also showed that increased accuracy with fewer oscillations for the Bleich-Sandler problem can be accomplished by simply reducing the amount of artificial damping used with the CAFE method. Because both the FE-FCT and the reduced damping CAFE models use linear finite elements rather than spectral elements to discretize the domain they offer a significant computational savings over CASE in these problems.

\section{Future work}

For future work, the current FE-FCT algorithm, which is applicable only to structure meshes, will be extended to multiple dimensions for application to more realistic UNDEX problems. Some details of this are presented for two-dimensional problems in [4,36]. The main challenge is in adapting the flux limiter in Xiao's FE-FCT algorithm to the multiple dimensional problems [4] and determining if the addition of the FCT algorithm still gives results comparable to CASE and increased computational efficiency for the multiple dimension case.

\section{References}

[1] ABAQUS Inc., ABAQUS v. 6.6 Benchmarks Manual, 2007.

[2] ABAQUS Inc., ABAQUS v. 6.6 Users Manual, 2007.

[3] H.H. Bleich and I.S. Sandler, Interaction Between Structures and Bilinear Fluids, International Journal of Solids and Structures 6 (1970), 617-639.

[4] D.L. Book, The Conception, Gestation, Birth, and Infancy of FCT, in: Flux-Corrected Transport: Principles, Algorithms, and Applications, D. Kuzmin, R. Lohner and S. Turek, eds, Springer, 2005, pp. 5-28.

[5] D.L. Book, J.P. Boris and K. Hain, Flux-Corrected Transport II: Generalizations of the Method, Journal of Computational Physics 18(3) (1975), 248-283.

[6] J.P. Boris and D.L. Book, Flux-Corrected Transport I. SHASTA, A fluid transport algorithm that works, Journal of Computational Physics 135 (1997), 172-186.

[7] J.P. Boris and D.L. Book, Flux-Corrected Transport. III. Minimal-Error FCT Algorithms, Journal of Computational Physics 20(4) (1976), $397-431$.

[8] M.A. Christon et al., A Mixed Time Integration Method for Large Scale Acoustic Fluid-Structure Interaction, CED-Vol. 6, High Performance Computing in Computational Dynamics, ASME, 1994, pp. 25-38.

[9] R.H. Cole, Underwater Explosions, Princeton University Press, 1948.

[10] J.A. DeRuntz, Application of the USA Code to Underwater Shock Problems, 73rd Shock and Vibration Symposium, 2002.

[11] C. Farhat, M. Lesoinne and P. LeTallec, Load and motion transfer algorithms for fluid/structure interaction problems with non-matching discrete interfaces: momentum and energy conservation, optimal discretization, and application to aeroelasticity, Computer Methods in Applied Mechanics and Engineering 157 (1998), 95-114.

[12] C.A. Felippa and J.A. DeRuntz, Acoustic fluid volume modeling by the displacement potential formulation, with emphasis on the wedge element, Computers \& Structures 41(4) (1991), 669-686.

[13] C.A. Felippa and J.A. DeRuntz, Finite element analysis of shock induced hull cavitation, Computer Methods in Applied Mechanics and Engineering 44 (1984), 297-337.

[14] G.E. Georghiou, R. Morrow and A.C. Metaxas, A two-dimensional, finite-element, flux-corrected transport algorithm for the solution of gas discharge problems, Journal of Physics D 33 (2000), 2453-2466.

[15] J. Giannakouros and G.E.M. Karniadakis, Spectral Element-FCT method for scalar hyperbolic conservation laws, International Journal for Numerical Methods in Fluids 14 (1992), 707-727.

[16] J. Giannakouros and G.E.M. Karniadakis, A spectral element-FCT method for the compressible Euler equations, Journal of Computational Physics 115 (1994), 65-85.

[17] D. Givoli, Numerical Methods for Problems in Infinite Domains, Elsevier, 1992.

[18] S.W. Gong and K.Y. Lam, On attenuation of floating structure response to underwater shock, International Journal of Impact Engineering 32 (2006), 1857-1877. 
[19] C.F. Hung, P.Y. Hsu and J.J. Hwang-Fuu, Elastic shock response of an air-backed plate to underwater explosion, International Journal of Impact Engineering 35 (2005), 151-168.

[20] L.E. Kinsler et al., Fundamentals of Acoustics - Fourth Edition, (4th ed.), Wiley, 2000.

[21] B. Klenow and A. Brown, Assessment of Non-Reflecting Boundary Conditions for Application in Far-Field UNDEX Finite Element Models, 77th Shock and Vibration Symposium, SAVIAC, Monterey, CA, 2006.

[22] T.G. Liu et al., Isentropic one-fluid modeling of unsteady cavitating flow, Journal of Computational Physics 201 (2004), 80-108.

[23] R. Lohner, K. Morgan, J. Peraire and M. Vahdati, Finite element flux-corrected transport (FEM-FCT) for the Euler and Navier-Stokes equations, International Journal for Numerical Methods in Fluids 7 (1987), 103-109.

[24] H. Mair et al., Lagrangian Hydrocode Modeling of Undewater Explosive/Target Interaction, 61st Shock and Vibration Symposium, 1990.

[25] W.A. Mulder, Spurious modes in finite-element discretizations of the wave equation may not be all that bad, Applied Numerical Mathematics 30 (1999), 425-445.

[26] R.E. Newton, Effects of Cavitation on Underwater Shock Loading - Part I, Naval Post Graduate School, Monterey, CA, 1978.

[27] R.E. Newton, Finite Element Analysis of Shock-Induced Cavitation, ASCE Spring Convention, Portland, OR, 1980.

[28] P.B.R. Nimmagadda and J. Cipolla, A pressure based cavitation model for underwater shock problems, 71st Shock and Vibration Symposium, 2001.

[29] C. Pozrikidis, Introduction to Finite and Spectral Element Methods using MATLAB, Chapman \& Hall/CRC, 2005.

[30] Y. Shin and L.D. Santiago, Surface ship shock modeling and simulation: two-dimensional analysis, Shock and Vibration 5 (1998), 129-137.

[31] Y. Shin and N. Schneider, Ship Shock Trial Simulation of USS Winston S. Churchill (DDG 81): Modeling and Simulation Strategy and Surrounding Fluid Volume Effects, 74th Shock and Vibration Symposium, 2003.

[32] Y.S. Shin, Total Ship Shock Modeling and Simulation Using LS-DYNA/USA, LS-DYNA User Conference 2000, Osaka, Japan, 2000.

[33] M.A. Sprague and T.L. Geers, Computational treatment of cavitation effects in near-free-surface underwater shock analysis, Shock and Vibration 8(2) (2001), 105-122.

[34] M.A. Sprague and T.L. Geers, A Spectral-Element Method for Modeling Cavitation in Transient Fluid-Structure Interaction, International Journal for Numerical Methods in Engineering 60 (2004), 2467-2499.

[35] M.A. Sprague and T.L. Geers, A Spectral-Element Method for Modeling Cavitation in Transient Fluid-Structure Interaction, Journal of Computational Physics 32 (2003), 1857-1877.

[36] M.A. Sprague and T.L. Geers, A spectral-element/finite-element analysis of a ship-like structure subjected to an underwater explosion, Computer Methods in Applied Mechanics and Engineering 195 (2006), 2149-2167.

[37] S.L. Wood, Cavitation Effects on a Ship-Like Box Structure Subjected to an Underwater Explosion, Naval Postgraduate School, 1996.

[38] S. Xiao, An FE-FCT method with implicit functions for the study of shock wave propagation in solids, Wave Motion 40 (2004), $263-276$.

[39] W.F. Xie et al., Application of a one-fluid model for large scale homogeneous unsteady cavitation: The modified Schmidt model, Computers \& Fluids 35 (2006), 1177-1192.

[40] W.F. Xie et al., Dynamic response of deformable structures subjected to shock load and cavitation reload, Computational Mechanics 40 (2007), 667-681

[41] S.T. Zalesak, The Design of Flux-Corrected Transport (FCT) Algorithms For Structured Grids, in: Flux-Corrected Transport: Principles, Algorithms, and Applications, D. Kuzmin, R. Lohner and S. Turek, ed., Springer, 2005, pp. 29-78.

[42] S.T. Zalesak, Fully Multidimensional Flux-Corrected Transport Algorithms for Fluids, Journal of Computational Physics 31(3) (1979), $335-362$.

[43] O.C. Zienkiewicz and P. Bettess, Fluid-Structre Dynamic Interaction and Wave Forces. An Introduction to Numerical Treatment, International Journal for Numerical Methods in Engineering 13 (1978), 1-16.

[44] O.C. Zienkiewicz, D.K. Paul and E. Hinton, Cavitation in Fluid-Structure Response (With Particular Reference to Dams Under Earthquake Loading), Earthquake Engineering and Structural Dynamics 11 (1983), 463-481. 

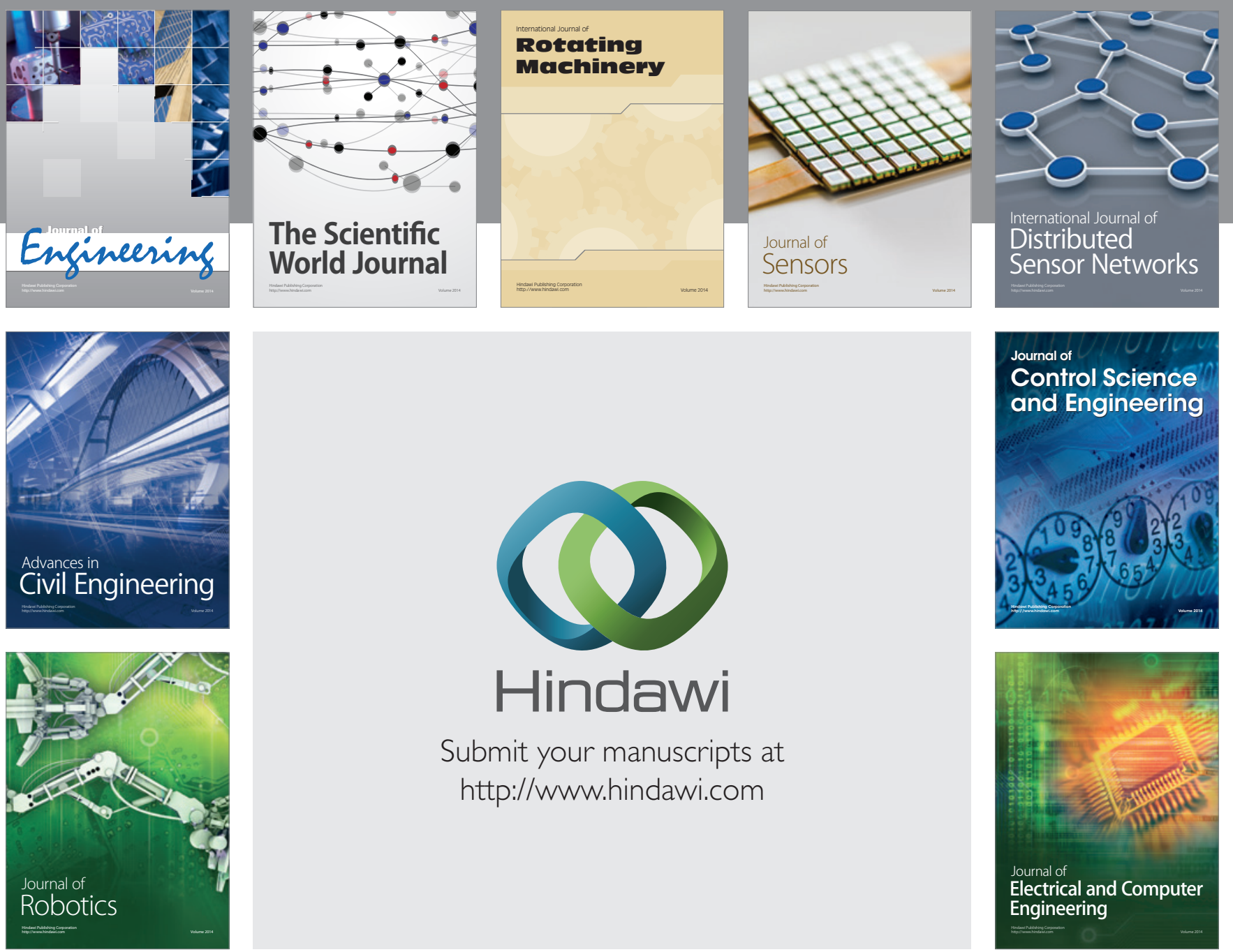

Submit your manuscripts at

http://www.hindawi.com
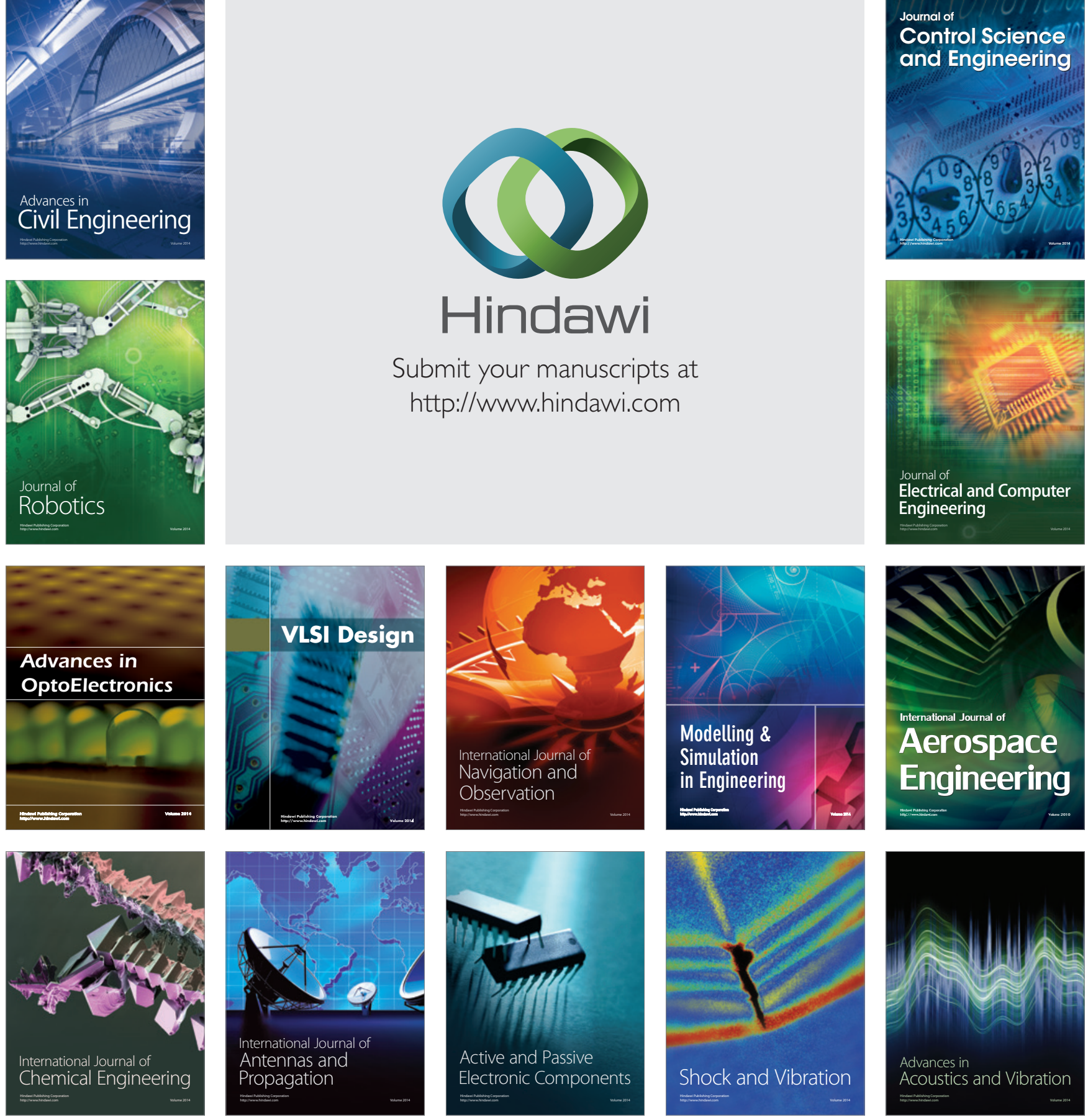